This is a non-peer-reviewed preprint submitted to EarthArXiv. The manuscript has been submitted for publication to the Mathematical Geoscience. 
Preprint- Moulaeifard et al. (Submitted to Mathematical Geoscince)

\title{
Subdivide and Conquer: Adapting Non-Manifold Subdivision Surfaces Method to Represent and Approximate Complex Geological and Reservoir Structures
}

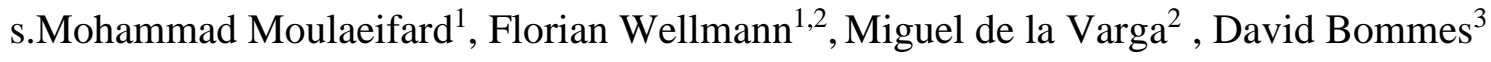 \\ ${ }^{1}$ Computational Geoscience and Reservoir Engineering, RWTH Aachen University, Aachen, Germany \\ ${ }^{2}$ Terranigma Solutions GmbH, Aachen, Germany \\ ${ }^{3}$ Computer Graphics Group, Institute of Computer Science, University of Bern, Bern, Switzerland \\ Correspond Author: s.Mohammad Moulaeifard (mohammad.moulaeifard@cgre.rwth-aachen.de)
}

\begin{abstract}
\end{abstract}
Computer graphics have gradually developed practical techniques to address models with the complex topology, in particular, by parametric surface-based modeling approach. Also, geologists have used this approach because it provides significant gains over grid-based modeling (e.g., implicit modeling) by using grid-free surfaces. However, since this approach originates from computer graphics, not all the capacities and limitations of this approach have been considered and investigated in geological modeling.

With this aim in mind, this paper investigates surface-based geological modeling through both geological and computer graphics approaches. NURBS (Non-Uniform Rational B-Splines) and subdivision surfaces, as two main parametric surface-based modeling methods, are investigated, and the strengths and weaknesses of both are compared. Although NURBS surfaces have been used in geological modeling, subdivision surfaces as a standard method in the animation and gaming industries, have received little attention in geological modeling. Subdivision surfaces support arbitrary topologies and watertight modeling, which are quite useful for complex geological modeling.

Investigating subdivision schemes with semi-sharp creases is an important part of this paper. Semi-sharp creases show the resistance of a mesh structure to the subdivision procedure, which provides a unique method for complex geological and reservoir modeling. Moreover, non-manifold topologies, as a challenging concept in complex geological and reservoir modeling, are explored, and the subdivision surfaces compatible with non-manifold topology are declared.

Finally, the approximation of complex geological structures by the non-manifold subdivision surface method is investigated with two different case studies. The approximated mesh is a simplified and less complex version of the original mesh while the important details of the original mesh are preserved. It not only significantly reduces the cost of modeling and simulation (by reducing the number of vertices to less 
than $5 \%$ of the number of vertices of the original mesh) but also, has features such as being watertight, smooth, topologically identical to the main original mesh and controllable with few control points.

Keywords Surface-based modeling. Subdivision surfaces. Non-manifold topology. Approximation of geological structures. Grid free. NURBS.

\section{Introduction}

Surface representation is one of the common concepts between geology and computer graphics. According to Botsch et al. (2010), implicit and parametric representations can be considered two main types of surface representations, where in both types, the surface is defined by a specific function; "implicit surfaces" are defined by a scalar-valued function and the aim is to find a zero level set on a 2D or 3D predefined grid, whereas a "parametric surface" is defined by a vector-valued function, and the aim is to convert the 3D models to 2D models in the paramedic domain. A parametric representation has significant gains over an implicit representation, as it can present details more compact and can be easily modified, although it has difficulty in the calculation of spatial queries (Botsch et al. 2010).

Similar to computer graphics, parametric surface-based geological and reservoir representations are defined by the surrounding surfaces (Jacquemyn et al. 2019; Wellmann and Caumon 2018; Graham et al. 2015a, b; Jackson et al. 2015, 2013; Deveugle et al. 2011; Caumon et al. 2009, De Kemp 1999). In contrast to gridbased implicit geomodeling, one of the significant advantages of parametric surface-based methods is that most of the important details of the model, such as heterogeneity, will be well maintained (Jacquemyn et al. 2019; Ruiu et al. 2016; Pyrczetal. 2009; Zhang et al. 2009). Additionally, the grid-based approach leads to problems in modeling formations, including faults, diaper flanks, folds, injected bodies and even various petrophysical features (Jacquemyn et al. 2019). In addition to implicit and parametric surface-based models, hybrid methods have been investigated in previous studies (Ruiu et al. 2016; Hassanpour et al. 2013; Pyrcz et al. 2009). Although hybrid approaches lead to more acceptable and faithful results, the requirement of a high-resolution grid cannot be neglected (Jacquemyn et al. 2019).

From a computer graphics point of view, spline surfaces and subdivision surfaces are two types of parametric surface-based representations (Botsch et al. 2010). Spline surfaces are the usual standard for computer-aided design (CAD), while subdivision surfaces are mostly used in computer gaming, animation and the film industry (Cashman 2010, Botsch et al. 2010). Generally, subdivision surfaces and NURBS both yield controllable freeform representations, but in different ways; NURBS emphasize the "smooth manipulation" of the model, whereas subdivision surfaces tend to release the model from "topological 
limitations (constraints)" (Cashman 2010) and enable surfaces with "arbitrary topology" (Botsch et al. 2010). The term topology refers to the connection between different elements of the model, and in geological modeling, it is a vital constraint for most geological procedures and actions, e.g., fluid flow, heat transfer and deformation (Thiele et al. 2016).

Jacquemyn et al. $(2019,2016)$ hold the view that using NURBS in geology and reservoir modeling has been limited until now because such modeling was originally based on grid-based modeling method. Previous studies using NURBS for geological, reservoir and fracture modeling showed that NURBS have been used for a variety of goals in this context (Jacquemyn et al. 2019, 2016; Börneretal. 2015; Zehner et al. 2015; Florez et al. 2014; Corbett et al. 2012; Geiger and Matthäi 2012; Caumon et al. 2009; Paluszny et al. 2007). However, subdivision surfaces have rarely been used in geological and reservoir modeling. Chen and Liu (2012) investigated geological modeling using the subdivision surface method. Although their work deserves appreciation as the first steps of using this method in explicit geological modeling, the authors did not explain the practical details of this approach and offered no explanation for the distinction between using spline surfaces and subdivision surfaces in parametric surface-based geological modeling, especially for non-manifold topology. The term 'Non-manifold' has been used to refer to structures that consist of multiple faces sharing one edge or multiple edges sharing one vertex (Chatzivasileiadi et al. 2018). These structures need more complex algorithms for the representations (Rossignac and Cardoze 1999). From the geological modeling point of view, the representation of contacts between geological interfaces when multiples faces of the mesh sharing one edge (e.g. intersection between faults or between faults and other layers) is a type of representation for non-manifold topology (Caumon et al. 2004). Also, complex geological structures commonly comprise multiple intersecting surfaces (Dassi et al. 2014). Therefore, nonmanifold topology is crucial in complex geological and reservoir modeling.

This work aims to contribute to complex geological and reservoir modeling by using non-manifold subdivision surfaces algorithm (surface-based geological modeling). Fig. 1 represent two different and common non-manifold geological structures represented by non-manifold subdivision surfaces algorithm in which their meshes consist of multiple faces shared one edge at the interfaces. In this paper, not only the limitations and advantages of subdivision surfaces and spline surfaces, as the two main parametric and gridfree methods, are investigated but also, non-manifold topology, as one of the challenges in complex modeling, is demonstrated and analyzed.

Additionally, the approximation of complex geological and reservoir structures with non-manifold subdivision surfaces is investigated. The approximated models are similar to the original models while 
Preprint- Moulaeifard et al. (Submitted to Mathematical Geoscince)

having less complexity which are suitable for processing goals (Ma et al. 2015). Approximated model by non-manifold subdivision surfaces exploited all advantages of surface-based modelling (e.g. being gridfree, smooth and controllable with some few numbers of control points). Also, using the approximated models for geological simulation can remarkably reduce the cost of processing by reducing the number of vertices. For better representation, the figures rendered by Blender, an open-source 3D computer graphics software (Community, B. O. 2018).

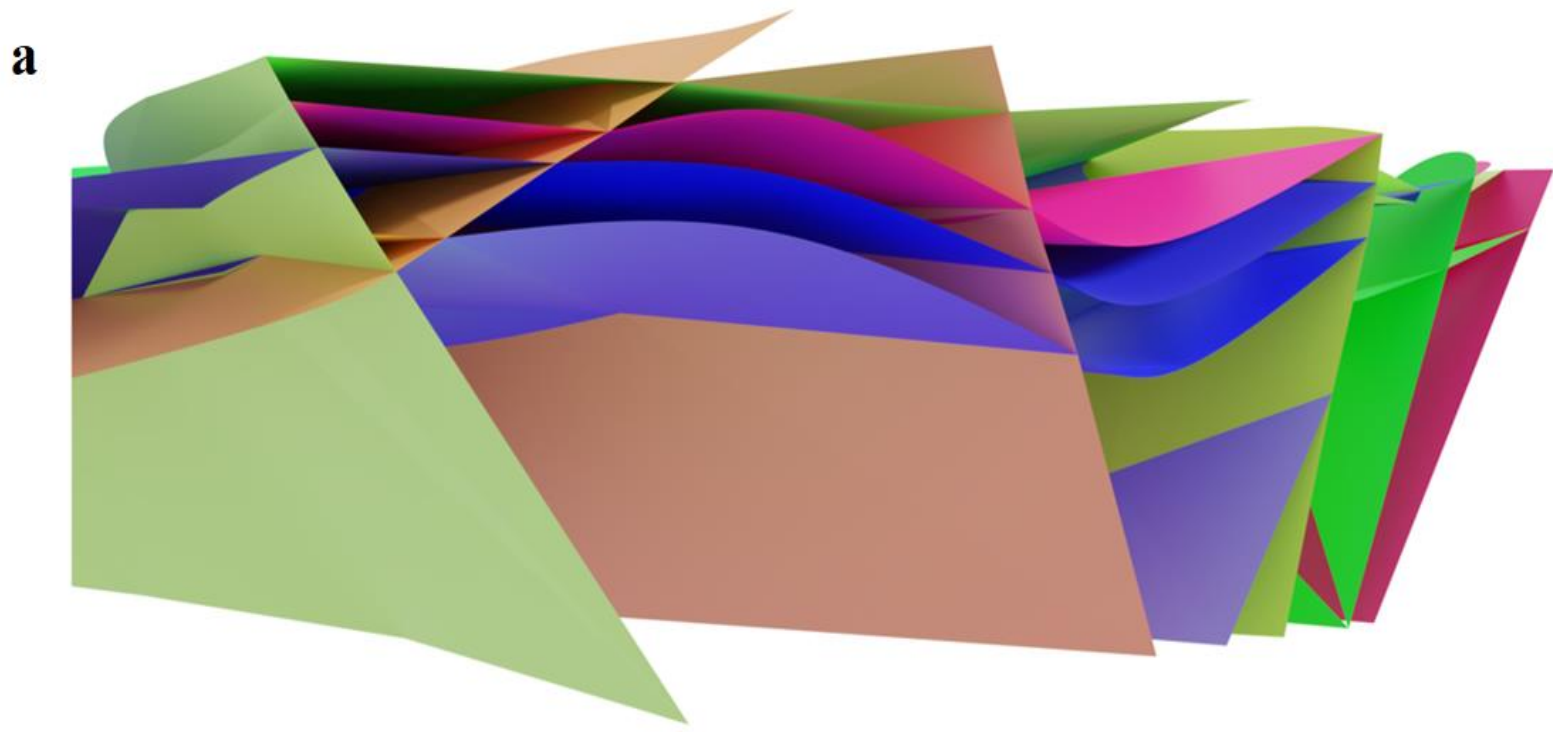

b

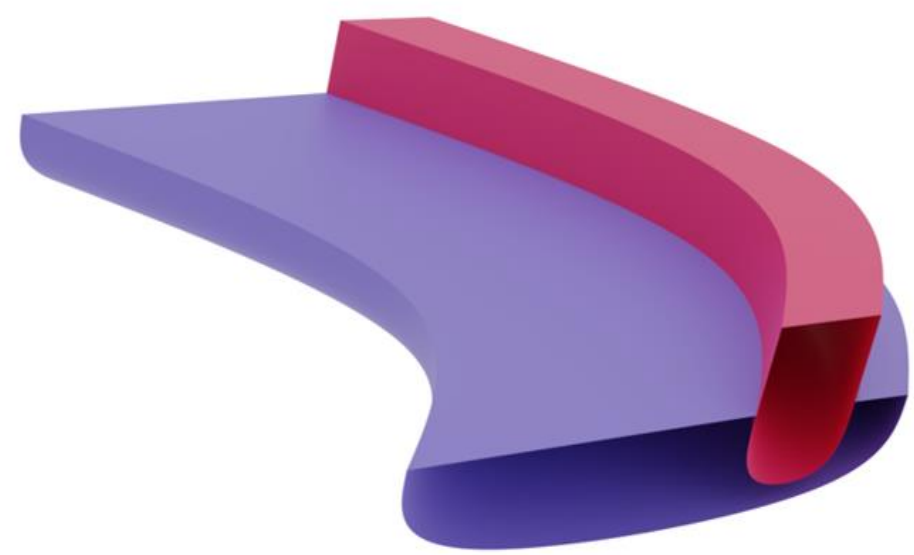

Fig. 1 Two examples of complex geological structures with non-manifold topologies represented by using non-manifold subdivision surfaces method a Geological structure consists of several faults which have intersections with other faults and geological layers. b Representation of two intersected channels. 
Preprint- Moulaeifard et al. (Submitted to Mathematical Geoscince)

\section{Methodology}

\subsection{Patches}

112 Generally, a parametric surface is created by a collection of different types of patches. Patches are made of 113 two significant parts: control points and the surfaces affected by the control points. The control points can 114 manage and control a triangular or rectangular 2D parameterized surface (in the parametric coordinates $U$ 115 and $\mathrm{V}$ ). By mapping the 2D parametrized surfaces to $3 \mathrm{D}$ coordinates (X, Y, Z), desirable smooth 3D 116 surfaces are created in $\mathrm{X}, \mathrm{Y}$ and $\mathrm{Z}$ coordinates (Fig. 2a). The control points and how they can affect the 117 surfaces play an important role in the representation of parametric surfaces (Fig. 2b). The control points 118 can impact the surfaces by different basis functions, which result in various types of patches, e.g., NURBS, 119 B-spline, Bezier, and triangulated patches.

$\mathbf{a}$
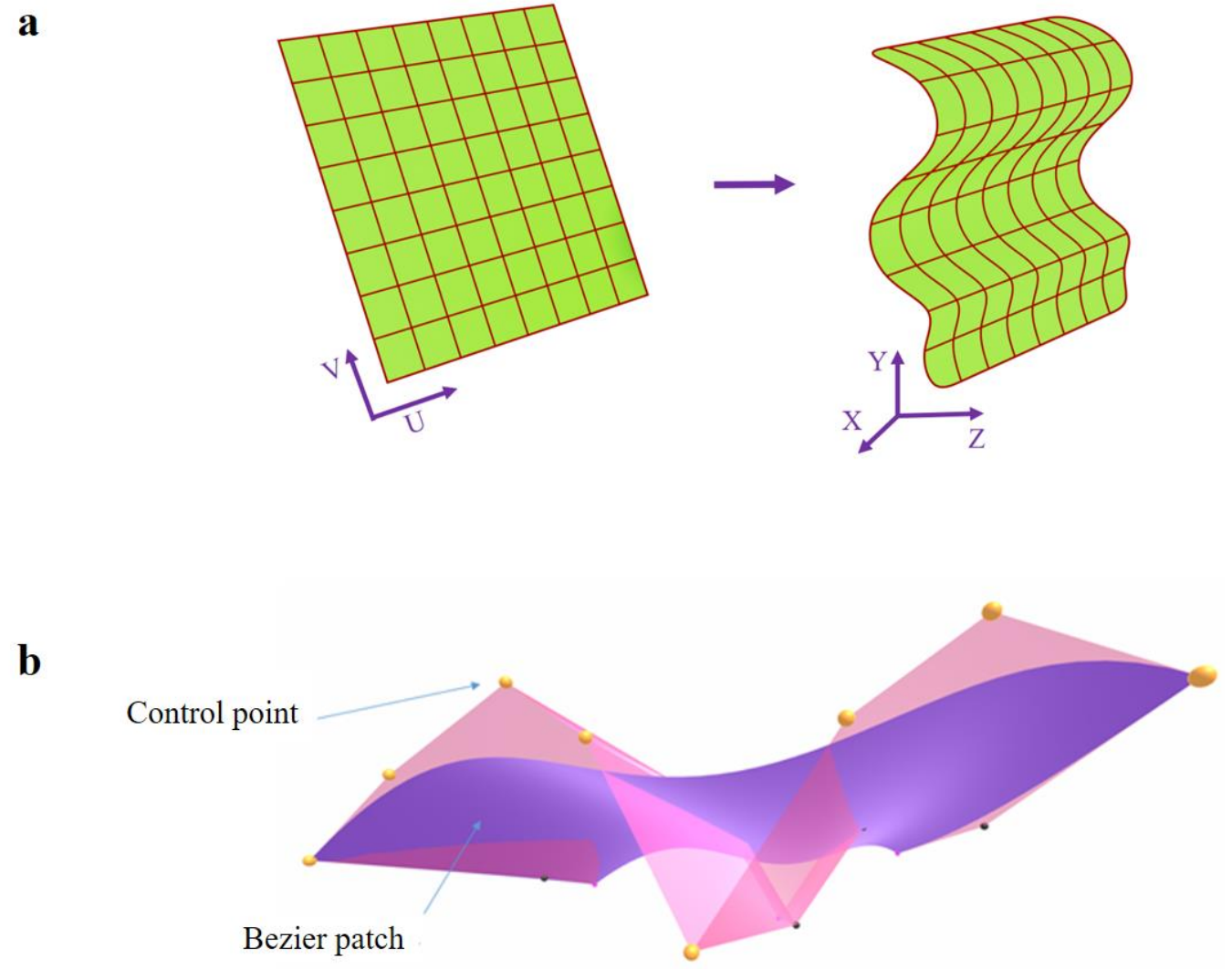

Fig. 2 a Mapping a rectangular 2D parametrized surface to 3D coordinates. b An example of a patch: a Bezier patch (purple surface) based on the control points (pink surface). 


\subsection{Piecewise parametric surfaces}

125 Studies on free-form surfaces are mostly based on parametric surfaces, which has resulted in modeling 126 developments with piecewise parametric surfaces (Sederberg 1985). Piecewise parametric surfaces, as an important tool in geometrical representation, are created by combinations of several patches. One of the common ways to build a set of patches of piecewise parametric surfaces is to use a rectangular grid of control points (Fig. 3). Changing the position of the control point(s) with specific basis function(s) can affect one or multiple patches and change the shape of the model. Importantly, the rectangular grid of piecewise parametric surfaces is different from the grid of implicit modeling.

$\mathbf{a}$

Rectangular grid of control points

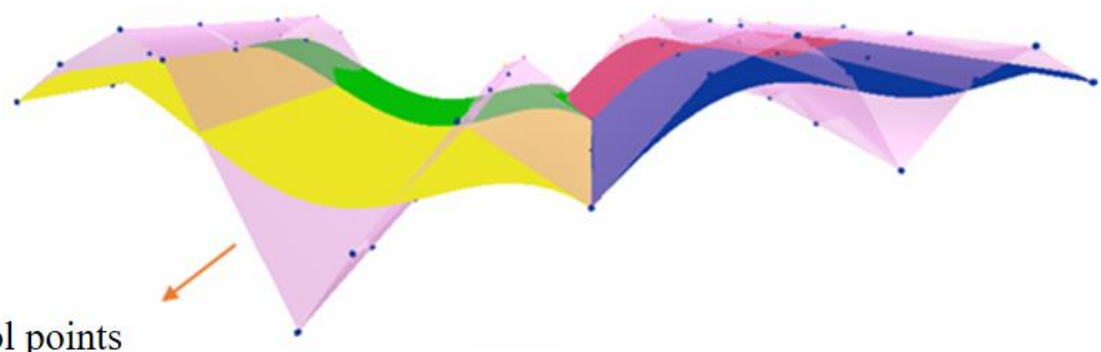

\section{b}

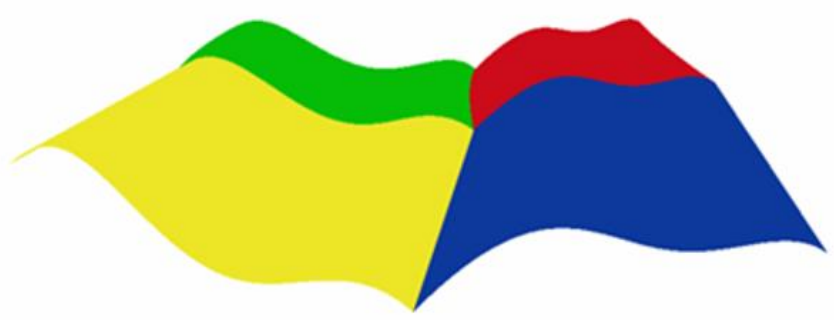

Fig. 3 An example of a piecewise parametric surface. a Piecewise Bezier surface beside a rectangular grid of control points (pink surface). b Piecewise Bezier surface with four Bezier patches.

\subsection{Spline Surfaces}

Spline surfaces are a type of piecewise parametric surface for creating high-quality free-form surfaces, which are produced by the smooth combination of several polynomial patches. Similar to piecewise parametric structures, spline surfaces are created by mapping from the rectangular parametric domain $(\mathrm{u}, \mathrm{v})$ to the $R^{3}(\mathrm{x}, \mathrm{y}, \mathrm{z})$ domain. A general surface can be obtained by 
141 where $c_{i j}$ are the control points in $R^{3}$ and $m+1$ and $k+1$ are the numbers of control points in the $u$ and $142 v$ directions, respectively. Additionally, $N_{i}^{n}(u)$ and $N_{i}^{n}(v)$ are spline blending functions in the $u$ and $v$ 143 directions, e.g., B-spline (basis spline) functions (Botsch et al. 2010).

144 NURBS surfaces are famous spline surfaces that are useful for making high-quality, freeform and editable surfaces (Botsch et al. 2010). Theoretically, NURBS surfaces are parametric surfaces that can be made according to the numbers of weighted points (control points), parametric knot vectors and specific interpolation degrees between the control points (Piegl and Tiller, 1997). NURBS (NonuNon-Uniform Rational B-Spline) surfaces have three important features, which are as follows: on basis spline functions. They include control points and the surface affected by the control points.

2) Rational: This means that the control points of the B-spline have weight values that can change the effect of a control point on a surface or, from a mathematical point of view, can affect the basis function associated with the control points.

154 Until now, NURBS have been considered combinations of B-spline patches near each other that have control points and specific basis functions.

3) Non-Uniform: This feature makes NURBS suitable for several practical goals (Cashman 2010). NURBS surfaces are combinations of polynaminal sections joined with each other at specific positions, which are knots (Cashman 2010)). The knots make a surface able to be locally modified while the surface remains smooth. This means that changing the position or the weight of any favourite control point can affect only the related part of the mesh (not the whole mesh) (Jacquemyn et al. 2019). If the knots are equally positioned, it is a uniform B-spline. Otherwise (if the knots are arbitrarily distributed), it is a Non-Uniform 162 B-Splines (NURBS) surface.

163 Since NURBS surfaces were originally produced by computer graphics scientists and have been used in several geological, reservoir and fracture models, it is necessary to investigate the limitations of NURBS from a computer graphics point of view. 


\subsection{Limitations of NURBS Surfaces from a Computer Graphics Point of View}

170

171

172

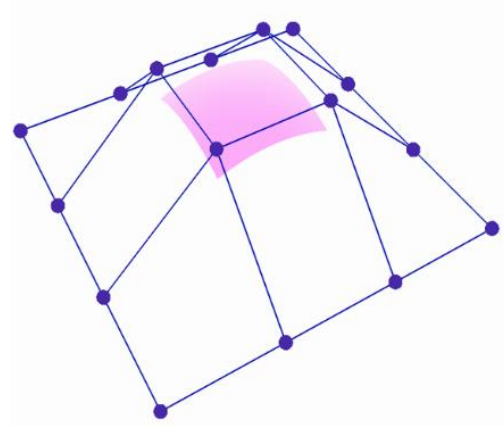

$\mathbf{a}$

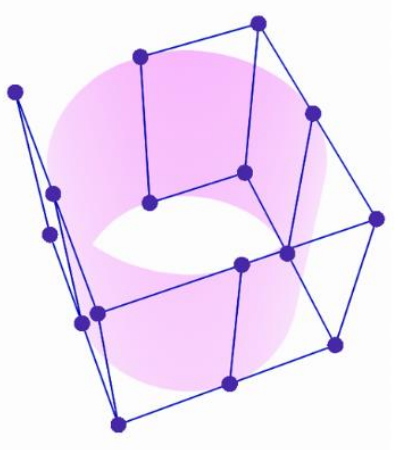

b

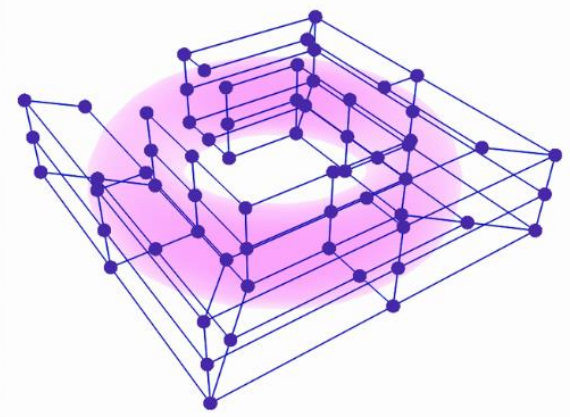

c
173

174

175

176

177

178

179

180

181

182

183

184

185

186

187

Fig. 4 Representation of the first limitation of NURBS surfaces: control points (blue) and NURBS surface (purple). Single NURBS surfaces are limited to surfaces that are topologically similar to a Sheet, $\mathbf{b}$ Cylinder or $\mathbf{c}$ Torus surfaces.

2- To create a model with complex topology, many NURBS patches should be smoothly connected (by stitching NURBS patches) (Fig. 5). Multiple connections between surface patches in addition to topological or geometrical constraints make the whole modeling procedure more complex (Bostch et al. 2010, Cashman 2010). As a result of the strict rectangular topology of NURBS surfaces, trimming the NURBS patches before stitching is fundamental during complex shape modeling, which can create unavoidable gaps between trimmed NURBS patches (Shen et al. 2014; Sederberg et al. 2008).

3- Modifying classical NURBS surfaces, e.g., adding more control points, will influence an entire row or column of control points (Botch et al. 2010). Indeed, preserving the grid structure of NURBS surfaces during local refinement is challenging (Fig. 6) (Derose et al. 1998). It should be mentioned that T-splines as a generalization of the NURBS, offer local refinement and can remarkably decrease the number of control points (Sederberg et al. 2004). 
Preprint- Moulaeifard et al. (Submitted to Mathematical Geoscince)

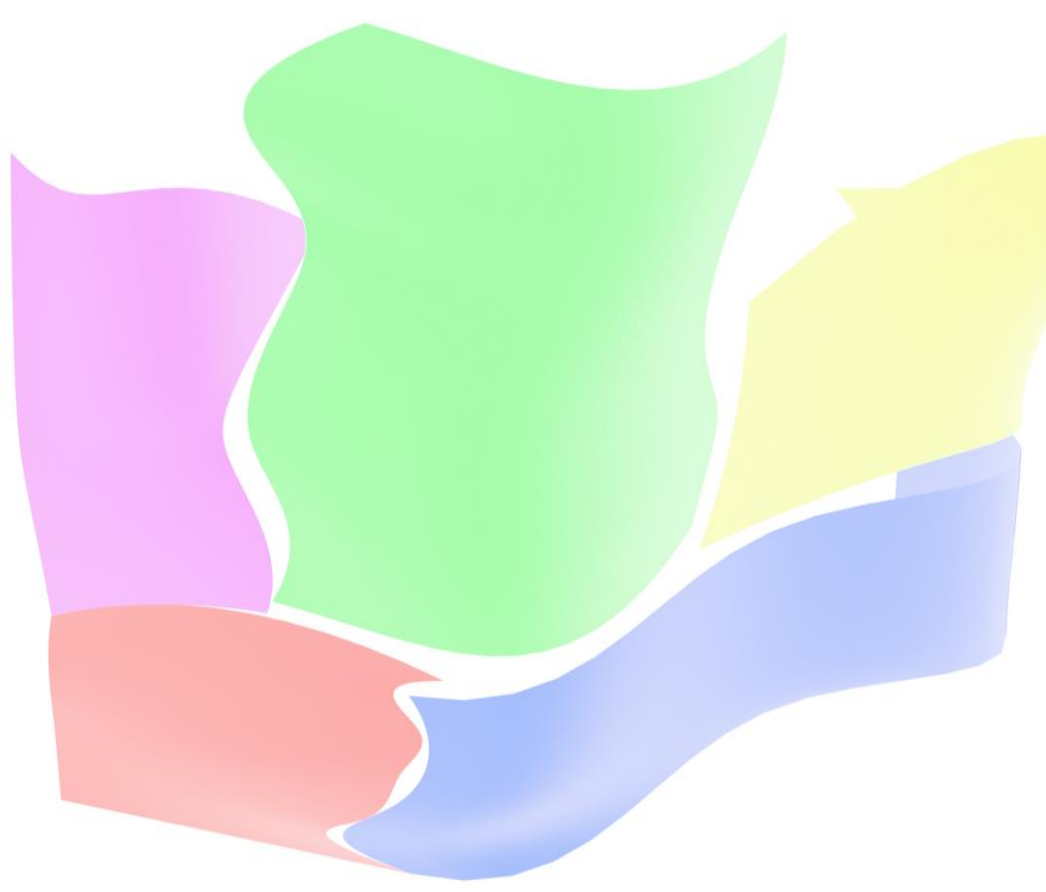

Fig. 5 Representation of the second limitation of NURBS surfaces: multiple NURBS patches should be stitched with others to build a complex structure. Additionally, trimming NURBS and keeping the final model smooth at the boundaries of patches is complicated (Derose et al. 1998).

$\mathbf{a}$

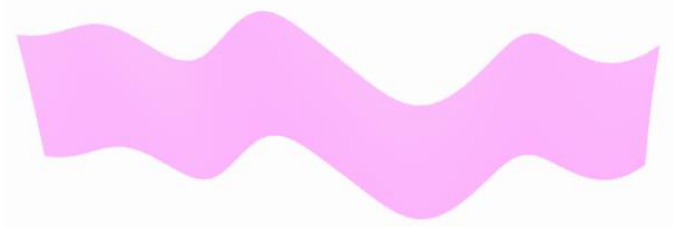

C

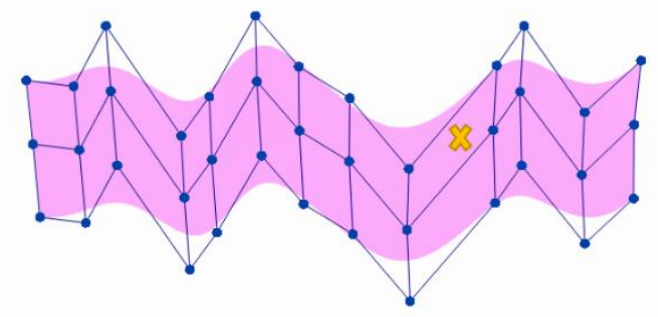

b

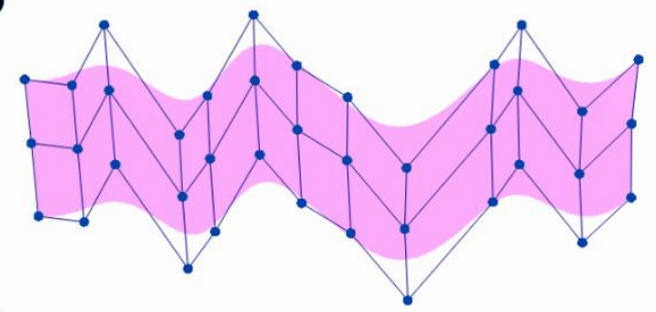

d

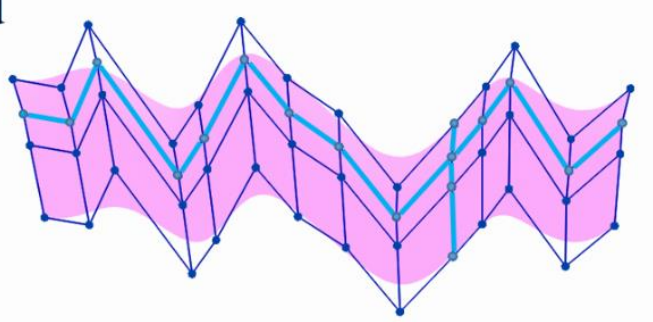

193 Fig. 6 Representation of the third limitation of NURBS surfaces: adding new control points affects the entire rectangular grid of control points. a Smooth NURBS surface with 39 control points. b Rectangular grid of control points (3 rows and 13 columns). c Considering the position of a new control point (yellow multiple). d Adding a new control point at a specific place increases the number of rows and columns to 4 and 14 , respectively. 


\subsection{Subdivision surfaces}

198

199

200

201

202

203

204

205

206

207

208

209

210

Fig. 7 An example of a simple subdivision surfaces method, converting a cube to a sphere by regularly applying the Catmull-

Using a grid of control points in piecewise parametric surfaces leads to topological constraints (Botsch et al. 2010). In complex models, topological limitations are more noticeable because complex models usually consist of different surfaces, and managing these surfaces, such as by "stitching" the surfaces together (for building watertight models) or trimming them (for editing), is complicated (Villemin et al. 2015). Therefore, complex representations require approaches that can support arbitrary topologies.

In essence, the subdivision scheme was created to overcome the difficulties of constructing smooth surfaces by arbitrary topology (Zorin and Schroder 2001, Catmull and Clark 1978, Doo and Sabin 1978). Subdivision surfaces can not only support arbitrary topology (in contrast with spline surfaces) but also be controlled by the control points of the mesh (similar to spline surfaces) (Botsch et al. 2010). Subdivision surfaces are mathematical instruments for repeated and converging implementations of rules for building smooth surfaces (Fig. 7). This method not only overcomes the limitations of NURBS by defining smooth and controllable surfaces that need no trimming for arbitrary topologies but is also computationally efficient and suitable for complex geometry (Zorin et al. 2000). To explain subdivision surfaces, first, basic concepts such as topology, mesh data (e.g., the positions of vertices) and shape should be clarified.
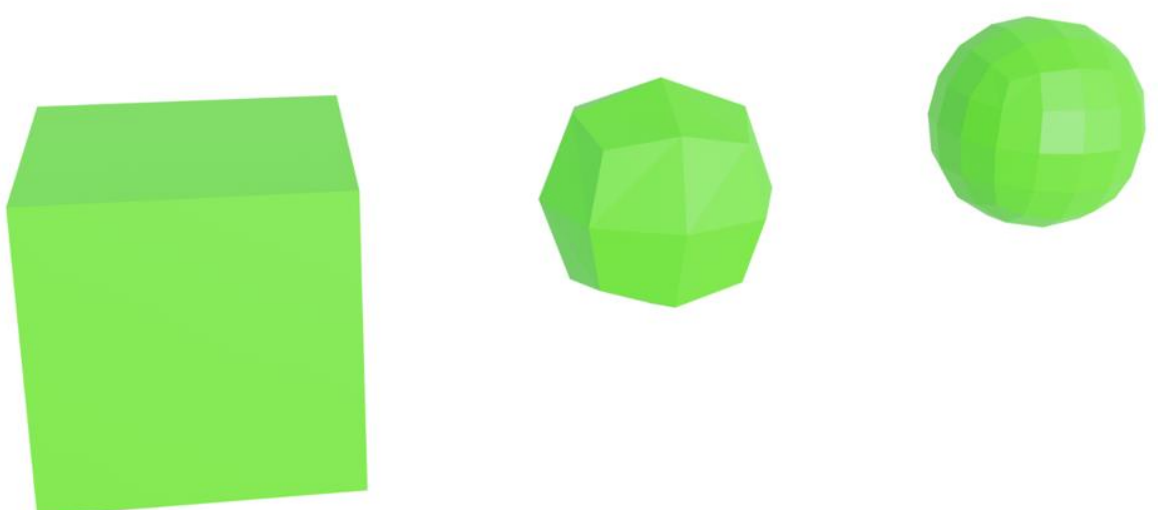

\section{a}

Clark subdivision scheme. a Original mesh. b \& c Apply one and two time (s) subdivision algorithm to the original mesh. 
Preprint- Moulaeifard et al. (Submitted to Mathematical Geoscince)

\subsection{Basics of subdivision surfaces: topology, mesh data and shape}

217 The shape of a model is basically a combination of the "topology" (connectivity) and "data" of the mesh of

218 the model (Fig. 8). The topology of the mesh represents the connections between the faces, edges and

219 vertices of the mesh, but the mesh data show the information related to the values associated with vertices,

220 faces and edges, such as the positions of the vertices. The distinction between the topology and mesh data

221 in producing the shape is necessary for the modeling of complex structures. Subdivision surfaces use the

222 positions of the vertices (mesh data) to create smooth surfaces by the regular iterative refinement of the

223 control vertices (Botch et al. 2010).

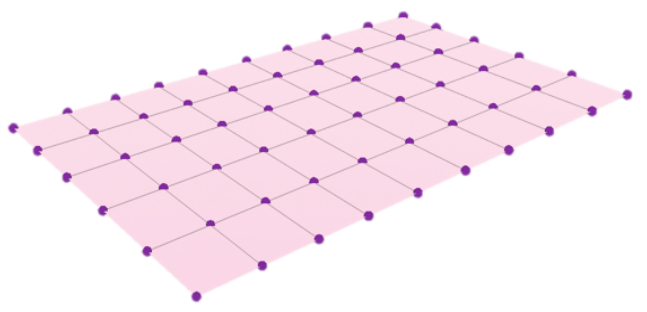

Mesh Data

\begin{tabular}{|c|}
\hline Vetex Numb \\
\hline 1 \\
\hline 2 \\
\hline 3 \\
\hline 4 \\
\hline 5 \\
\hline 6 \\
\hline
\end{tabular}

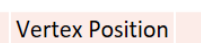

$$
\text { (X1,Y1,Z1) }
$$
$(X 2, Y 2, Z 2)$ (X3,Y3,Z3) (X4,Y4,Z4) $(X 5, Y 5, Z 5)$ $(X 6, Y 6, Z 6)$

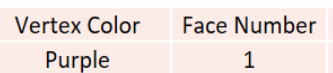

Face Norma
(N1,N2,N3)
(N4,N5,N6) Purple Purple

Purple

Purple

Purple (N7,N8,N9) (N10,N11,N12) (N13,N14,N15) (N16,N17,N18)

Face Color
Pink
Pink
Pink
Pink
Pink
Pink

Fig. 8 A shape is a combination of topology and mesh data. 


\subsection{Subdivision surface schemes and the subdivision zoo}

229 There are different variations of subdivision schemes, but they can be classified by important criteria, such 230 as the type of the original mesh (quadrilateral or triangular), the general rule for refinement and whether 231 the approach is based on approximation or interpolation.

232 Schröder and Zorin (2000) noted that during the subdivision surfaces procedure, either faces can be split into subfaces (primal), or vertices can be divided into multiple vertices (dual). In primal schemes, new vertices are created based on either interpolation or approximation of the original vertices, which divides this approach into two relevant categories: interpolation and approximation schemes.

236 In each refinement, if the original points of the initial surface are also points of the final (smooth) surface

237 and the positions of new vertices are defined based on interpolation between the original ones, it is an 238 interpolation approach, e.g., a modified butterfly approach. Otherwise, it is an approximation approach and 239 mathematically approximates the positions of "all" vertices (old and new vertices) to build a smooth shape; examples include the Catmull-Clark, Loop and $\sqrt{3}$ subdivision scheme approaches.

241 Two of the most common subdivision schemes are Loop and Catmull-Clark, which are based on the 242 approximate approach and generate triangular and quadrilateral meshes, respectively (Fabri and Pion 2009).

243 In this work, these two methods are used. Since both of these methods are based on an approximation 244 approach, it is necessary to know the basics of these methods. Approximation approaches generate smooth 245 curves or surfaces in two steps. First, new vertices are generated based on the position of the old vertices 246 (generation step), and second, the positions of the old vertices are changed (updated) based on the positions 247 of the new vertices by approximation rules (approximation step).

248 For example, one of the well-known approximation methods for building a smooth curve or surface is the 249 cubic B-Spline approximation. In each refinement of this approximation, first, new vertices are generated 250 precisely in the middle of each edge (Fig. 9a). In the second step, the new positions of the old vertices are 251 approximated by a weighted combination of the old and new vertices (Fig. 9b). 
Preprint- Moulaeifard et al. (Submitted to Mathematical Geoscince)

$\mathbf{a}$

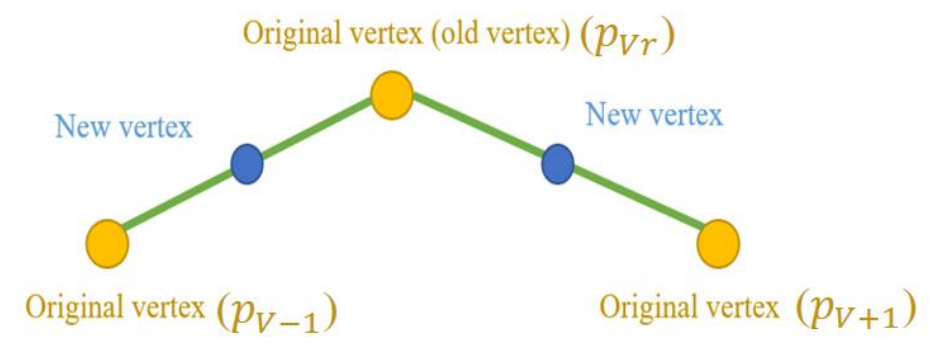

b

Original vertex (old vertex)

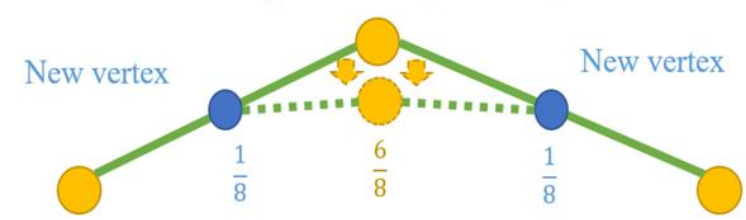

Original vertex

Original vertex

Fig. 9 a The first approximation step; the new vertices (blue) are produced in the middle of the edge. b The second

255 The new position of the old vertex $\left(p_{V}\right)$, which is between the positions of two adjacent new vertices $p_{V-1}$

256 and $p_{V+1}$, is determined by

$257 \quad p_{V}=\frac{1}{8} * p_{V-1}+\frac{1}{8} * p_{V+1}+\frac{6}{8} * p_{V}$,

258 To achieve the desired smoothness of the curve, the approximation procedure (refinement) should be 259 repeated; e.g., Fig. 10 shows two refinements of one curve with 8 control points. 


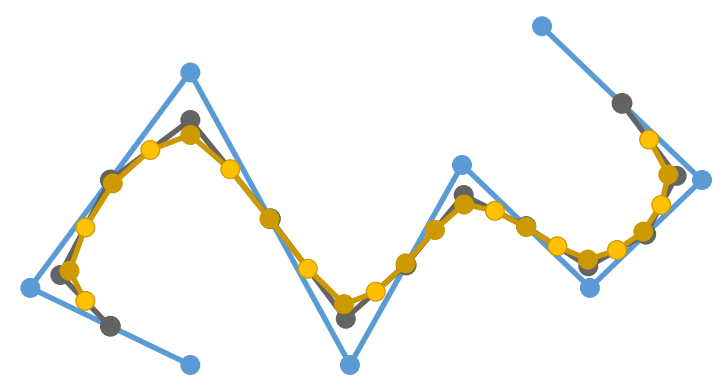

Fig. 10 A smooth curve with 8 control points after two refinements (the blue, black and brown lines are the original line, single refinement and double refinements, respectively)

\subsubsection{Loop Subdivision Scheme}

265 The Loop scheme, defined by Charles Loop (1987), builds smooth surfaces based on triangle meshes by 266 using approximation approaches. Similar to the cubic B-spline approximation approach, this scheme has

267 two steps in each refinement. In the first step, a new vertex $v$ should be generated on each edge, which can 268 be an interior or boundary edge (Fig. 11).

\section{$\mathbf{a}$}

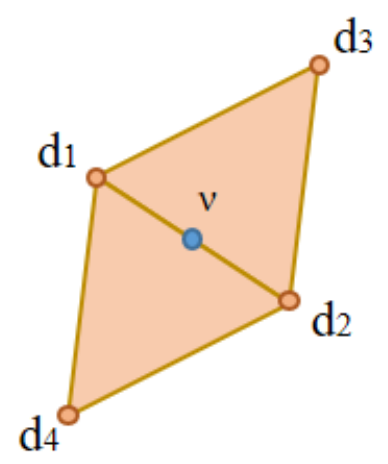

b

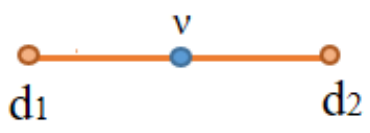

Fig. 11 Defining the new vertex $v$ in the Loop scheme. $\mathbf{a} v$ is on the interior edge; $\mathbf{b} v$ is on the boundary edge.

271 The position of the new vertex $v$, which is on an interior or boundary edge, can be computed by 
Preprint- Moulaeifard et al. (Submitted to Mathematical Geoscince)

$272 v=\frac{3}{8}\left(d_{1}+d_{2}\right)+\frac{1}{8}\left(d_{3}+d_{4}\right)$,

$273 v=\frac{1}{2}\left(d_{1}+d_{2}\right)$,

$274 \quad(4)$

275 In the second step, the new positions of the original (old) vertices are computed (Fig. 12). If the original

276 vertex is interior and there are $k$ adjunct vertices around it, the new position of $v$ is determined by

$277 \quad v_{\text {new }}=v *(1-k \beta)+\beta \sum_{1}^{k} P$,

$278 \quad$ where $\beta=\frac{1}{n}\left(\frac{5}{8}-\left(\frac{3}{8}+\frac{1}{4} \cos \frac{2 \pi}{n}\right)^{2}\right)$,

279

280 If the old vertex $v$ is a boundary vertex and the two neighborhood vertices are $\mathrm{P}_{1}$ and $\mathrm{P}_{2}$, the new position 281 of $v$ can be determined by

282

$$
v_{\text {new }}=v * \frac{3}{4}+\left(\mathrm{P}_{1}+\mathrm{P}_{2}\right) * \frac{1}{8}
$$

$\mathbf{a}$

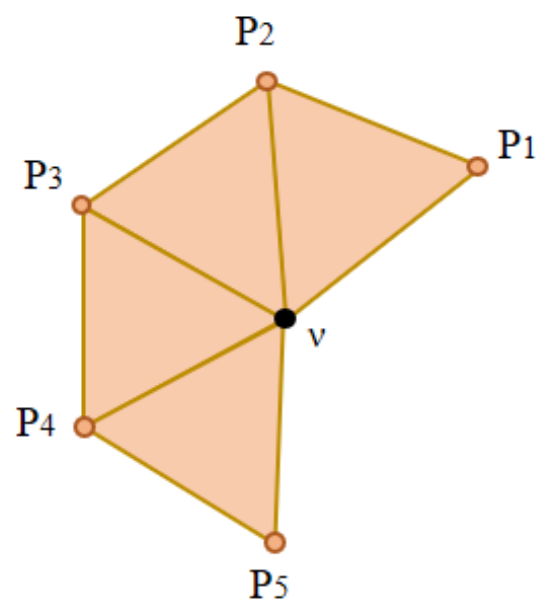

b

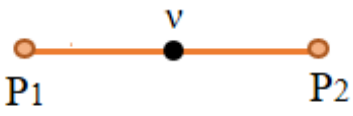

Fig. 12 Defining the positions of the old vertices in the Loop scheme: a the interior vertex. $\mathbf{b}$ the boundary vertex 
Preprint- Moulaeifard et al. (Submitted to Mathematical Geoscince)

\subsubsection{Catmull-Clark Subdivision Scheme}

287 The Catmull-Clark algorithm was first defined in 1978 by Edwin Catmull and Jim Clark (Catmull and Clark

288 1978). This scheme is a type of approximation approach and can be applied to polygonal meshes. Similar 289 to the other approximation-based methods, this scheme follows two steps: first, generate new vertices, and 290 then compute the new positions of the old vertices from the new vertices from the previous step.

291 For the boundary vertices, the algorithm is similar to the Loop scheme in both steps (cubic spline algorithm). 292 Generating the new vertices includes two parts: first, create a face point for each face (f), and second, make 293 an edge point $(e)$ on each interior edge (Fig. 13).

294 1) Each face has a face point (f)

$295 \mathrm{f}=\frac{1}{4} \sum_{k=1}^{4} \mathrm{~d}_{4}$,

296 2) Each interior edge has an edge point $(e)$

297

$e=\frac{1}{16}\left(d_{5}+d_{6}+6 * d_{7}+6 * d_{8}+d_{9}+d_{10}\right)$

298 In the second step, the new position of $v$ based on the face points and edge points around $v$, which are $\mathrm{f}_{i}$ 299 and $\mathrm{e}_{i}$, can be determined by

$300 \quad v_{\text {new }}=\frac{n-3}{n} * v+\frac{2}{n} * L+\frac{1}{n} * T$,

301 where $n$ is the number of face points or edge points around $v$ and

$302 L=\frac{1}{n} \sum_{i=1}^{n} \mathrm{e}_{i}$,

$303 \quad T=\frac{1}{n} \sum_{i=1}^{n} \mathrm{f}_{i}$, 
a

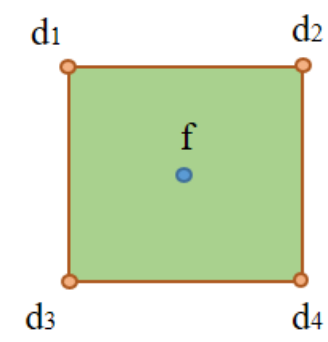

b

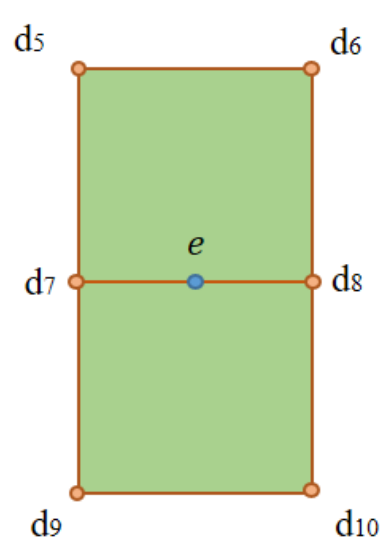

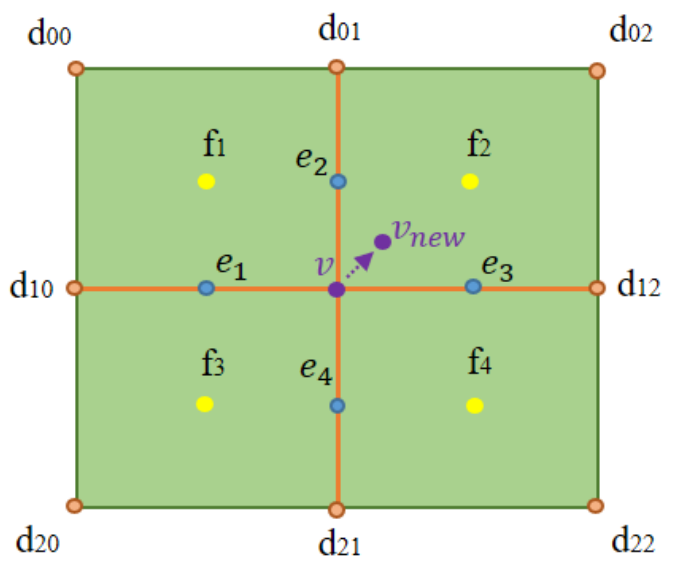

Fig. 13 Catmull-Clark subdivision scheme. a Finding the face point for each face. b Finding the edge point for each interior edge. c Computing the new position of vertex $v$ based on the neighborhood face and edge points.

\subsection{Subdivision Surfaces with Semi-Sharp Creases, A Tool for Complex Modeling}

Modifying the classical subdivision algorithm allows smooth surfaces to have sharp features such as creases and corners (Derose et al. 2000, Hoppe et al. 1994). Although real-world models such as geological structures do not have entirely sharp features, the ability to manage and control the sharpness of creases and corners during the subdivision procedure can be very useful in building complex structures. A crease can be created on the mesh by changing the mesh shape (e.g., by applying subdivision approaches or pulling the mesh) while pinching the specific vertices or edges of the mesh (Fig. 14). With more freedom given to the related vertices or edges, the sharpness of the crease decreases (semi-sharp crease). 
a
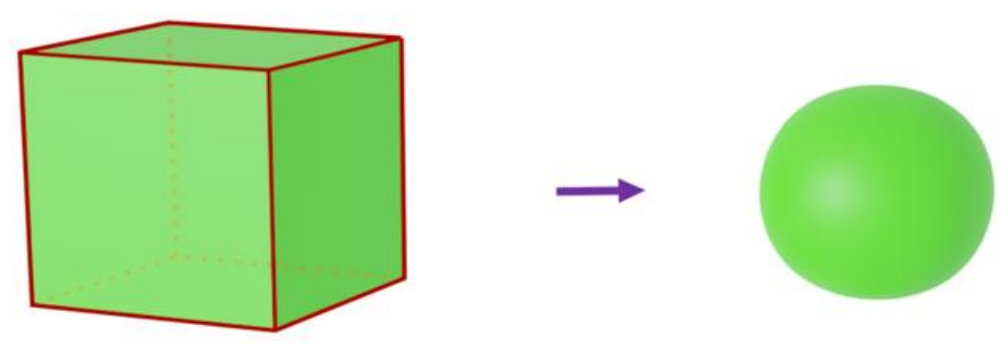

b
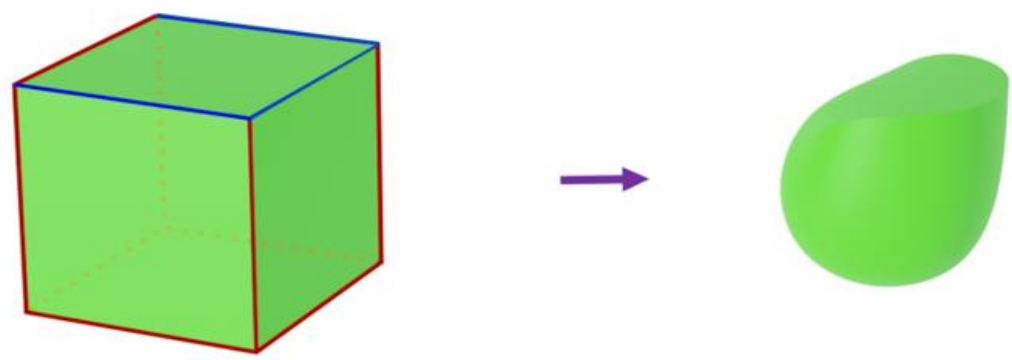

Fig. 14 Creating creases on a mesh by applying five times subdivision surfaces algorithm on the cube. a All edges of the cube are smooth edges (red edges). b Three edges are crease edges (blue edges), and nine edges are smooth (red edges).

319 Practically, during the subdivision of surfaces, it is possible to consider the average crease sharpness value 320 for each edge of the mesh. These numbers can show the resistance of the vertices of the edges to mesh 321 modification algorithms, e.g., resistance to smoothing by subdivision surfaces (if more than one edge is 322 connected to the vertex, the average value should be considered). The higher the crease sharpness value is, 323 the sharper the crease. This value can be between zero and infinite while zero indicates a smooth crease 324 (Derose et al. 2000).

\subsection{Subdivision surfaces compatible with non-manifold topologies}

327 Classical subdivision surfaces cannot support nan-manifold shapes. However, they can support both 328 watertight modeling and arbitrary topologies. Therefore, it is worthwhile to make changes in the procedure 329 of the classical subdivision surfaces method to make it supportive of non-manifold shapes. 
In the field of computer graphics and animation, non-manifold topology (NMT) is an important and challenging concept that includes a broad range of definitions (Ying and Zorin 2001). Additionally, in geological and reservoir modeling, non-manifold topologies are widely used in complex structures. Caumon et al. 2004 presented examples of non-manifold geological modeling, e.g., interactions between faults or faults with horizons by radial edge deltaic deposit reservoir models. In reservoir modeling, a connection between channels can be considered a non-manifold structure. One example of a surface with non-manifold topology is a surface that has several patches allocated to one boundary (Ying and Zorin 2001). Pixar Graphics Technologies (https://graphics.pixar.com) gives the most applicable definition of non-manifold topologies, which explains most cases. The definition is as follows:

Assume that one person is standing on one of the faces of the shape and wants to walk around each vertex. The person should start walking from the corner of any selected face and walk on all of the faces around the vertex that have the same normal orientation, then try to walk to the next unvisited face and repeat the procedure (Fig. 15). The mesh is a manifold if one of the following two cases occurs:

1) If the vertex is an interior vertex, the person arrived back at the starting point, and during this trip, the person visited all of the faces and edges around the vertex.

2) If the vertex is a boundary vertex, the person started at a boundary edge around the vertex and arrived at another boundary edge, and again, the person visited all the faces and edges around the vertex.

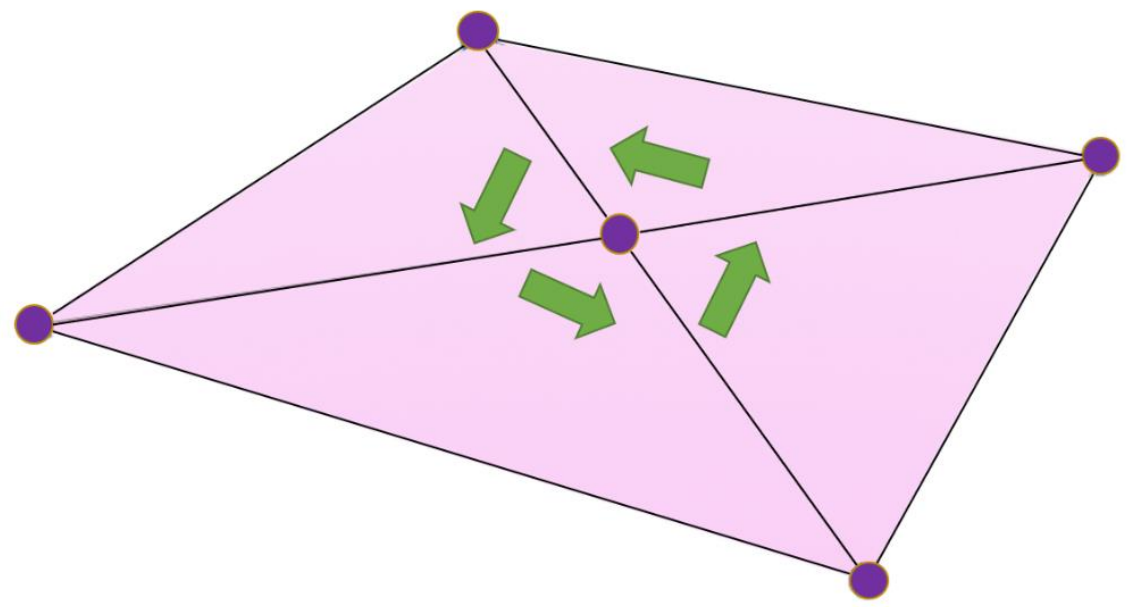

Fig. 15 An example of walking around the vertex 
Fig. 16 shows one of the common non-manifold shapes based on the definition above, in which three different faces share one edge. In the non-manifold structures, there is at least one local geodesic neighborhood, which makes the topology challenging and incompatible with many methods, such as subdivision surfaces (Botsch et al. 2010). However, the support for arbitrary topologies and the other excellent features of subdivision surfaces make it worthwhile to use modified subdivision surfaces for nonmanifold geological topologies.

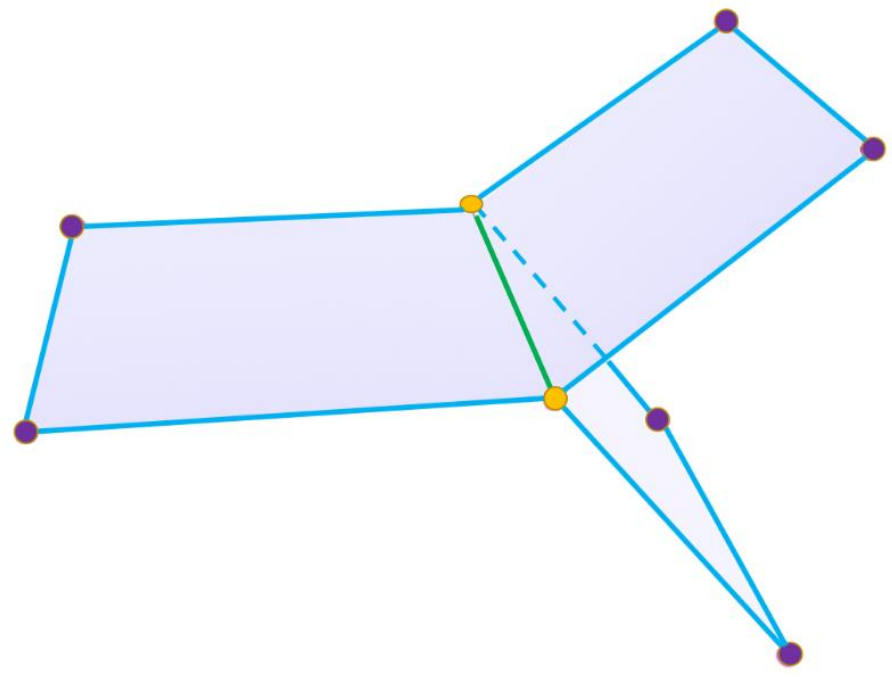

Fig. 16 Three faces share one edge; a typical example of non-manifold shape. Non-manifold vertices (yellow) and edge (green)

In complex geological structures, it is often unavoidable to encounter non-manifold structures because surfaces usually share common boundaries. On the other hand, the classic subdivision surfaces algorithm 363 can be applied to manifold meshes and produce manifold structures. The "Non-manifold subdivision 364 algorithm" makes it possible to receive the advantages of subdivision surfaces method such as arbitrary 365 topology and produce watertight volumes for non-manifold shapes. This algorithm defined by Ying and 366 Zorin 2001 and includes several detailed rules and covers a wide range of non-manifold problems in 367 computer graphics. In this paper, the rules that are related to the most common and general geological and 368 reservoir issues are explained. They defined the extended Loop subdivision algorithm to model nonmanifold structures, which is as follows: 
$\mathrm{T}(\mathrm{v})$ is considered the set of all triangles of the mesh around vertex v (Fig. 17). Based on the definition in the previous section, vertex $\mathrm{v}$ is a manifold vertex if two favourite sequential tringles are inside $\mathrm{T}(\mathrm{v})$ and

372 share one edge connected to v. This vertex can be either inside (interior vertex) or a boundary vertex.

373 Additionally, an edge is named a manifold edge if it is shared by two triangles of the mesh (the manifold 374 edge can be part of just one triangle if the edge is a boundary edge).

a

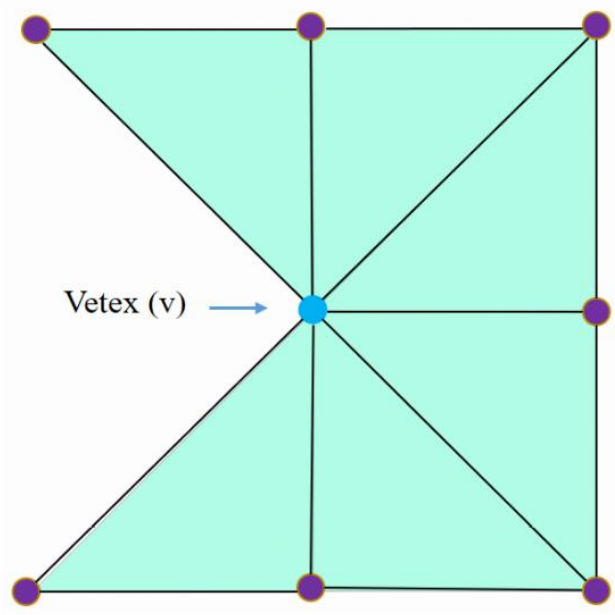

b

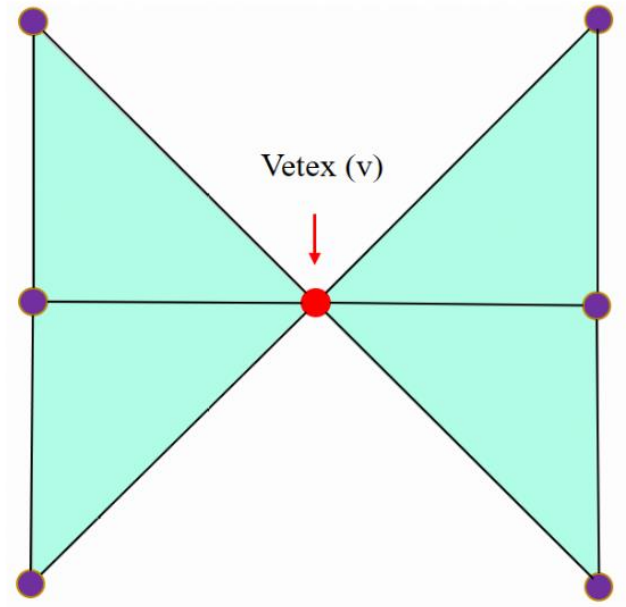

375

376

377

378

379

380

381

382

383

384

385

386

387 388

Fig. 17 Representation of T(v) (a set of triangles) around vertex v (center vertex). a Representation of a manifold vertex v (blue vertex); two favourite sequential tringles inside $\mathrm{T}(\mathrm{v})$ share one edge connected to $\mathrm{v}$. b Representation of a non-manifold vertex $\mathrm{v}$ (red vertex).

A non-manifold vertex and edge are named a singular vertex and edge, respectively. Considering M(v) as the largest set of triangles inside $\mathrm{T}(\mathrm{v})$, it consists of the specific triangles such that every pair of favourite sequential triangles around $\mathrm{v}$ share an edge (Fig. 18 shows the set of triangles $\mathrm{T}(\mathrm{v})$, which consist of $\mathrm{M}_{1}(\mathrm{v})$ and $\left.\mathrm{M}_{2}(\mathrm{v})\right)$.

It should be mentioned that the sets of triangles inside each $\mathrm{M}(\mathrm{v})$ can be either manifold or non-manifold. Also, non-manifold sets of triangles can be split into manifold sets. Therefore, each M(v) can be considered a combination of manifold segments, which are called $\mathrm{Q}(\mathrm{v})$; e.g., $\mathrm{M}_{1}(\mathrm{v})$ and $\mathrm{M}_{2}(\mathrm{v})$ consist of one and three $\mathrm{Q}(\mathrm{v})$, respectively. Indeed, $\mathrm{Q}(\mathrm{v})$ (the manifold set of triangles around $\mathrm{v}$ ) is the largest set of triangles such that all two sequential triangles of it share a manifold edge. 


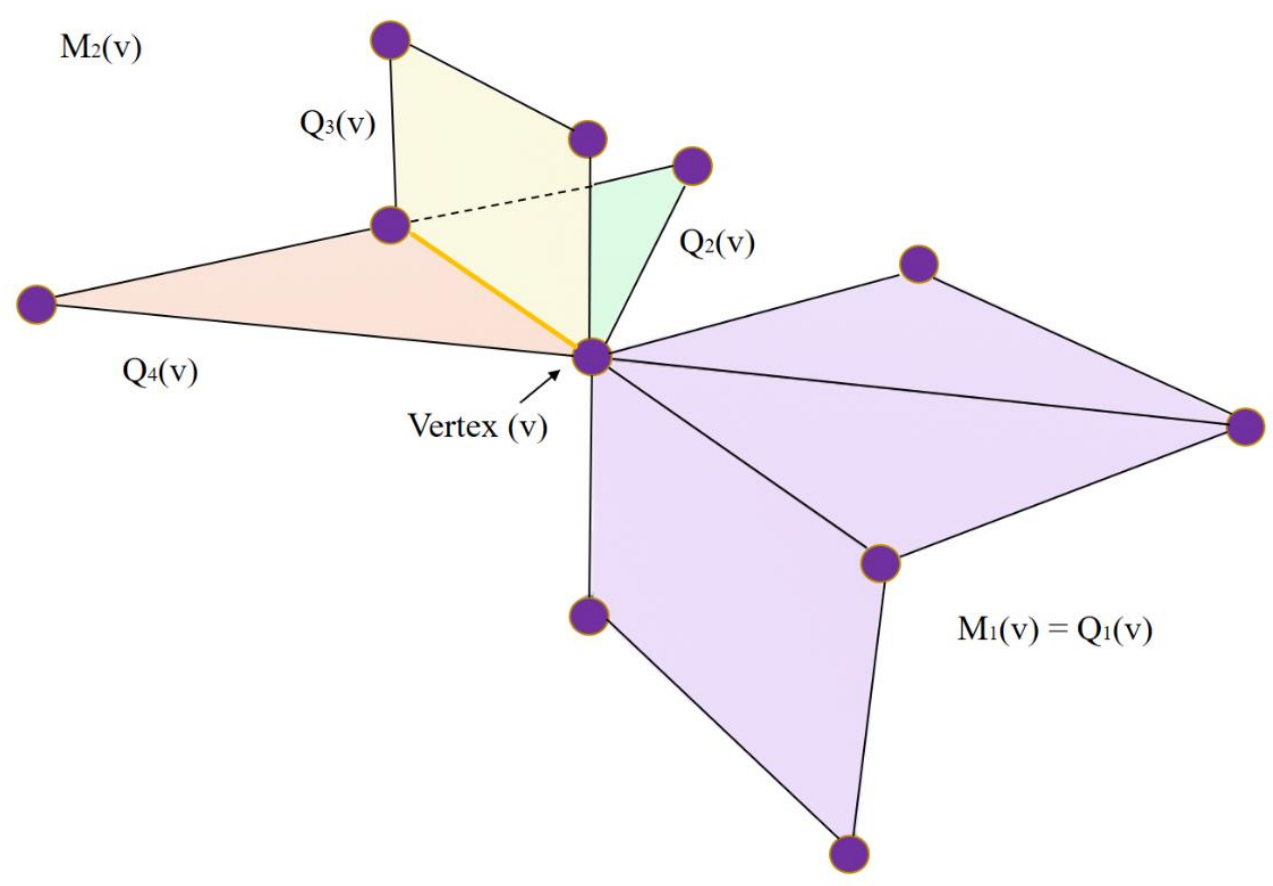

Fig. $18 \mathrm{~T}(\mathrm{v})$ consists of two parts; $\mathrm{M}_{2}(\mathrm{v})$ includes three manifold parts, $\mathrm{Q}_{2}(\mathrm{v}), \mathrm{Q}_{3}(\mathrm{v})$ and $\mathrm{Q} 4(\mathrm{v})$, and $\mathrm{M}_{1}(\mathrm{v})$ has one manifold part, $\mathrm{Q}_{1}(\mathrm{v})$. The yellow edge represents the non-manifold edge which is shared between three edges.

393 The singular vertex $\mathrm{v}$ is "simple" when it is part of a single $\mathrm{M}(\mathrm{v})$, and two singular edges should meet each 394 other at v (all of the Q(v)-manifold regions around v share edges); otherwise, it is a "complex" singular vertex (Fig. 19). For regular vertices, the standard Loop algorithm should be used. If the vertex is simple singular, the cubic B-spline subdivision algorithm (as mentioned previously) should be used. Otherwise,

397 the vertex is complex singular, and in most cases, a vertex can be fixed (the position will not be changed).

398 Additionally, if the edge is singular, it should be subdivided at the midpoint, and if it is not singular, it 399 should generally follow the regular Loop algorithm. For the other specific cases, please see Yian and Zorin 400 (2001). Fig. 1 shows two common and general examples of using non-manifold subdivision scheme in 401 geological and reservoir modeling. 


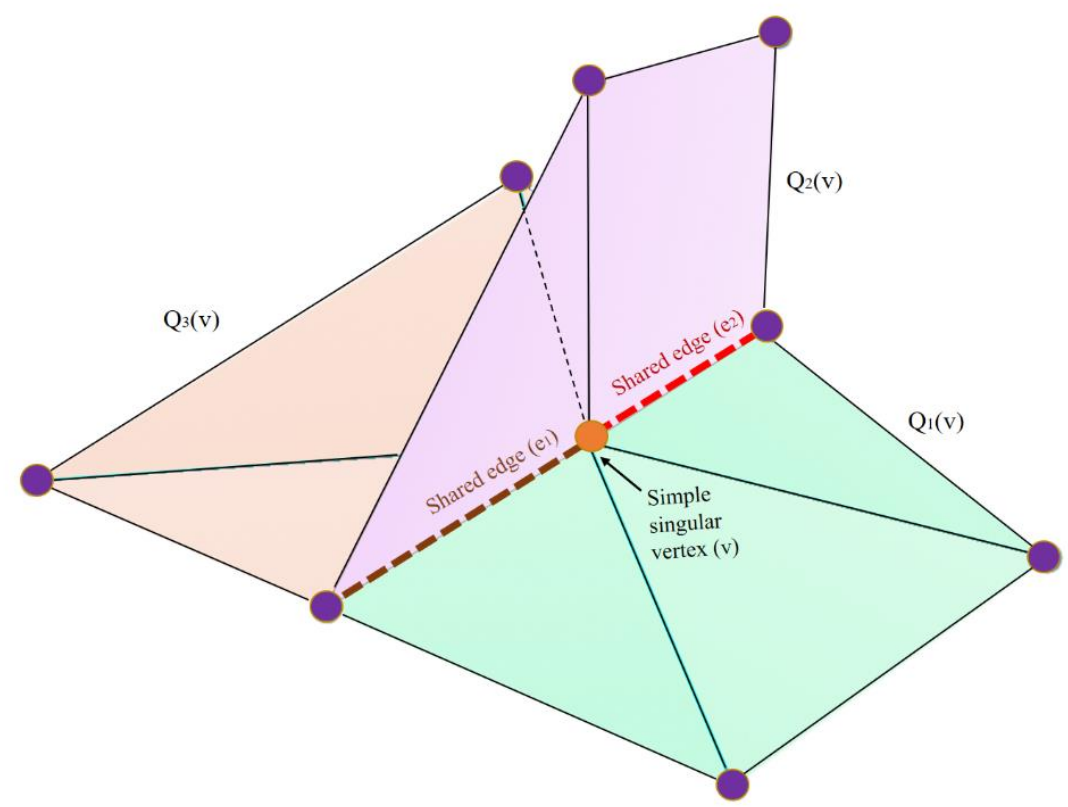

Fig. 19 Simple singular vertex (v) (orange vertex)

\section{Parametric Surface-based Geological Modeling}

Jacquemyn et al. 2019 defined geological domains as closed volumes, which are mostly limited by interacting surfaces. These surfaces not only must represent the correct topology of the geological model but also should have a watertight relationship with other surfaces. To build such closed volumes with NURBS, different NURBS surfaces (patches) are needed that can interact with each other in different ways (Jacquemyn et al. 2019). On the other hand, generally, the relationships between independent NURBS surfaces violate geological principles, and we need to consider approaches for remedying this, such as building parametric surfaces for the whole domain and modifying the model by trimming, cutting or extrapolating the surfaces (Wellmann and Caumon 2018). As mentioned previously, according to several computer graphics references (Botsch et al. 2010, Cashman 2010, Derose et al. 1998), the need for connecting, trimming and stitching different NURBS patches to each other to build a complex model is one of the limitations of NURBS. However, the necessity for stitching and trimming separate surface patches to make watertight closed-volume surfaces is eliminated in the subdivision surfaces approach by building surfaces and volumes with arbitrary topology (Cashman 2010). 
Preprint- Moulaeifard et al. (Submitted to Mathematical Geoscince)

To build surface-based geological structures by subdivision surfaces, we propose the following steps:

1- A seamless and arbitrary-topology mesh similar to the desired shape (mother mesh) is created. If the mesh contains layers, a one layered seamless topology should be defined.

2- Based on the final goal, the sharpness of the crease of each edge (crease sharpness value) is specified (understanding the edges and vertices that should try to resist during classical smoothing can help in this step).

3- The subdivision algorithm is applied based on the crease sharpness value of each edge.

4- If needed, the model is edited by changing the positions of the control points or the crease sharpness values of the edges to reach the final goal.

\subsection{Different types of geological surface interactions}

There are three different types of geological surface interactions that result in geological domains (Jacquemyn et al. 2019).

\subsubsection{Creating closed volumes by joining surfaces at their edges}

In this case, there are at least two surfaces that should be connected exactly on their edges (boundaries) to make a watertight volume (e.g., sinuous channels) (Fig. 20). Jacquemyn et al. 2019 explained how to use NURBS to build these complex shapes (Fig. 20a). In their work, two different surfaces that have exactly same edge geometries should be connected to each other. However, as mentioned previously, NURBS have a problem when patches must be stitched to each other (Botch et al. 2010).

Although Jacquemyn et al. 2019 mentioned solutions such as using the degree elevation procedure or adding more control points (which is one of the limitations of classical NURBS) and Ruiu et al. 2016 suggested to increase the multiplicity of the knots (which results in reduced continuity (Cashman 2010)), using subdivision surfaces method has fewer difficulties because of its inherent features, such as supporting arbitrary topology and watertight modeling.

To build similar closed volumes based on the subdivision surfaces method first, the seamless and arbitrary topological mesh of the model is defined (Fig. 20b). Having a seamless mesh at the first step of modeling will leave no concerns related to watertight modeling. In the second step, the crease sharpness values of all edges are specified. For example, in the sinuous channel case, because the top face of the channel is flat, most edges of the top face should fully resist during the subdivision procedure, and their crease sharpness values should be maximal and infinite, e.g., ten (blue edges). The other edges should be smoothly subdivided; therefore, their crease sharpness values are zero (red edges). 
$\mathbf{a}$
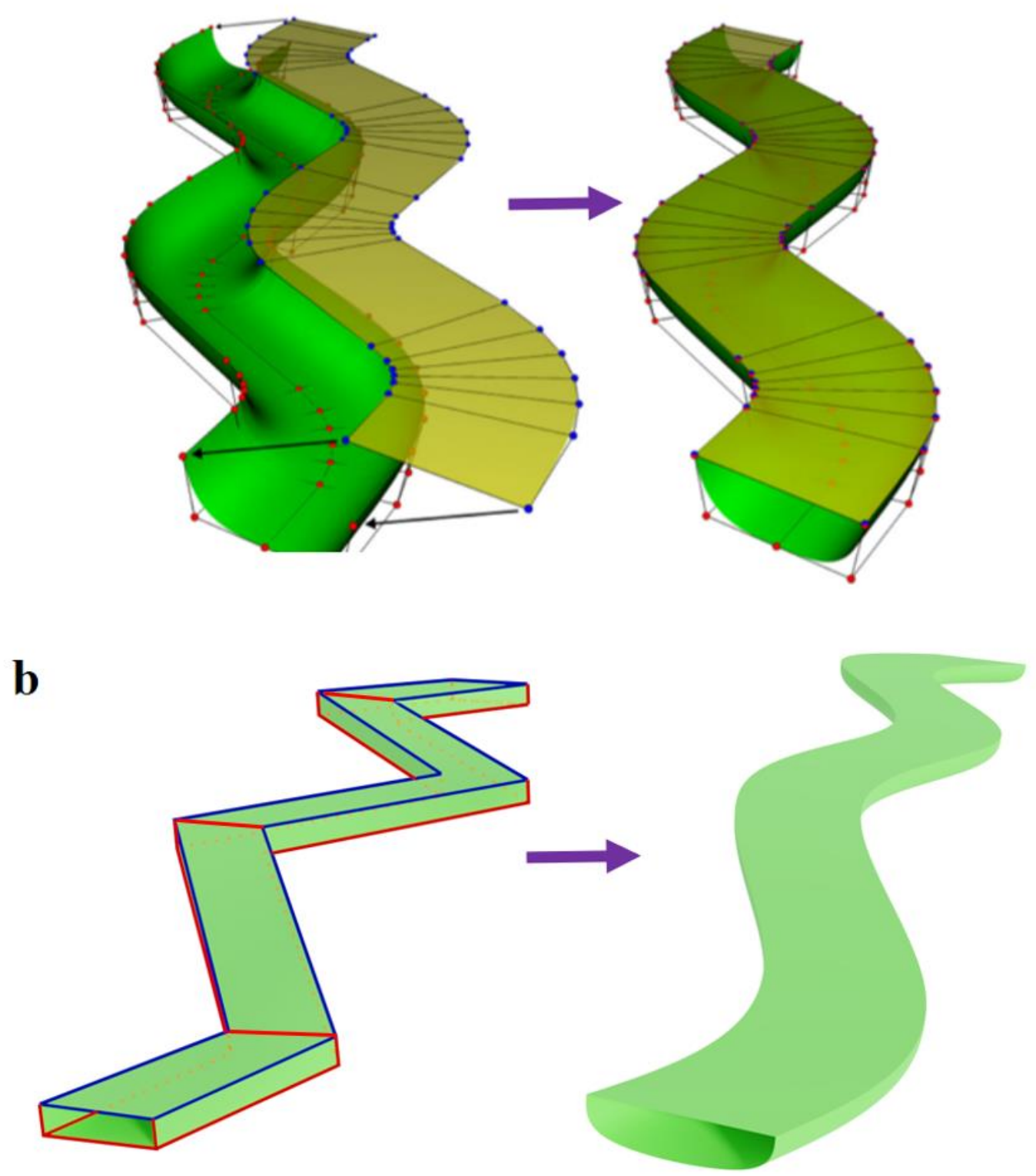

451

452 Fig. 20 Building watertight channels by NURBS (Jacquemyn et al. 2019) and subdivision surfaces (our approach). a Using 453 NURBS to join surfaces at their edges to create closed volumes. b Building a channel by the subdivision surfaces algorithm. The 454 crease sharpness value for each red and blue edge is zero and ten respectively. 
In the third step, the subdivision algorithm based on the crease sharpness value of each edge is applied.

457 The subdivision surfaces approach result gives a watertight and smooth channel, which can be controlled 458 by the control points.

\subsubsection{Distorted (warped) surfaces}

460 Warped geological structures can be considered a kind of complex geological formations and are observed in nature in different ways, as described below.

\subsubsection{Warped geological surfaces made by geological phenomena such as folding and faulting}

In these cases, the surfaces are irregularly made by faulting or folding, which poses challenges in geological modeling. Since the abilities of the selected method for modeling, such as the flexibility and consistency of structures (supporting arbitrary topologies), can play an important role in the whole modeling process, using subdivision surfaces instead of NURBS can lead to fewer difficulties, especially in layered warped structures. Fig. 21 shows a model of a faulted fold created by Catmull-Clark subdivision surfaces. Due to the suggested subdivision surfaces algorithm, first, the arbitrary topology of the whole mesh (two sperate cages) is defined. In the next step, the sharpness of the crease of each edge is assigned (the blue and red edges have crease sharpness values equal to ten and zero, respectively). Finally, the subdivision surfaces algorithm based on the crease sharpness value is applied.

$\mathbf{a}$

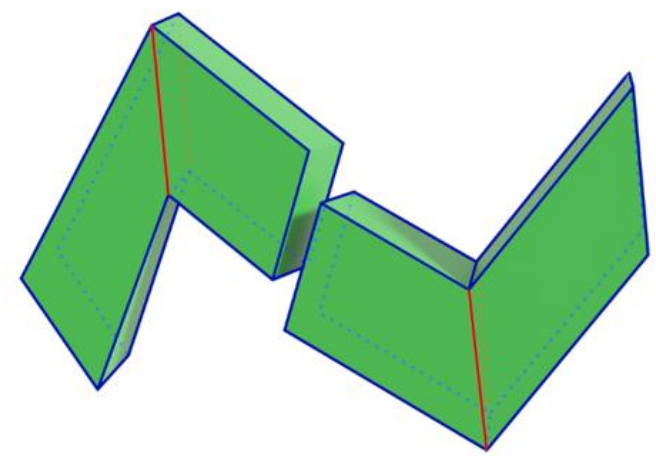

b

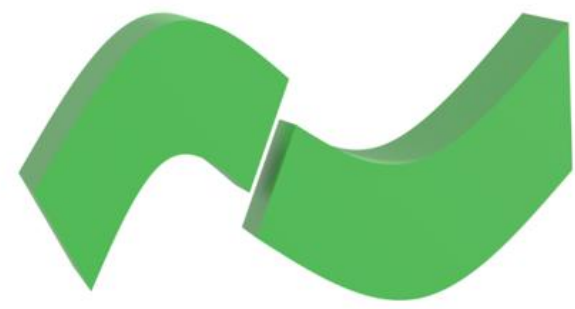




\subsubsection{Warped geological surfaces associated with other surfaces that have geometrical connections} with them

478 Such structures can be considered combinations of at least two NURBS surfaces with different grid 479 structures that should be matched to each other (by warping one of the surfaces) to make the new structure. 480 Jacquemyn et al. 2019 defined a procedure for building such structures based on NURBS. In their method, 481 the positions of the control points of the surface to be warped should be adapted to the parent surface(s) 482 (Fig. 22a). However, this adaption can be expensive due to the limitations of NURBS, such as difficulties in adding more control points (as mentioned before, this is only possible by splitting parameter intervals that affect an entire row or column of the control mesh (Botch et al. 2010)) and problems in trimming.
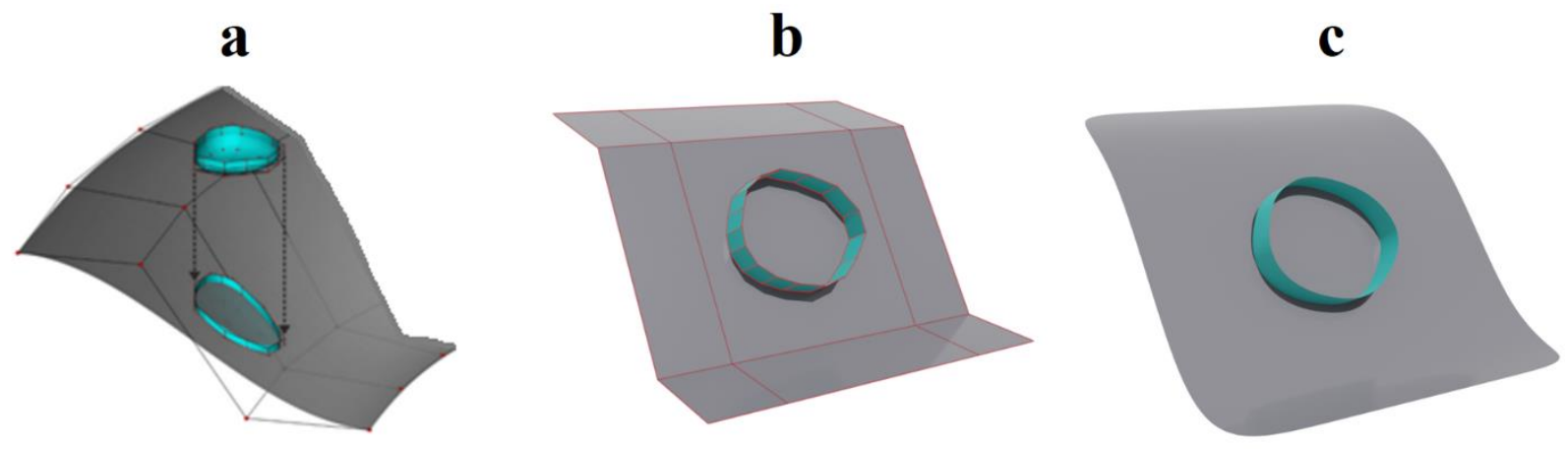

\section{d}

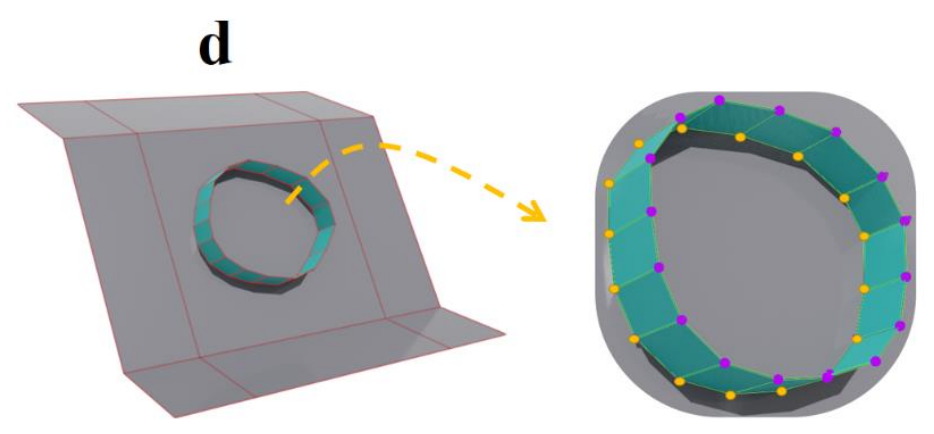

Fig. 22 Warped surfaces associated with other surfaces by: a NURBS warping of the bounding surface (blue) to conform to the geometry of a clinoform surface (gray) (Jacquemyn et al. 2019). b, c An example of using the subdivision surfaces algorithm for topologies; $\mathbf{c}$ The final shape obtained by assigning different crease sharpness values to each edge and applying the subdivision algorithm. d The bounding surface has a watertight connection with the clinoform surface (gray) by the shared vertices (yellow).

491 Subdivision surfaces approach, unlike NURBS, first consider one comprehensive topology consisting of a watertight structure for both surface topologies together, the warped and parent topologies, (instead of two 
separate topologies) (Fig. 22b) and then intelligently refine the model by assigning a specific crease sharpness value to each edge and apply the subdivision algorithm (Fig. 22c). Therefore, due to the limitation of NURBS, it is necessary to use surface-based modeling methods that can support arbitrary topologies 496 (e.g., subdivision surfaces) rather than grid-based parametric structures (e.g., NURBS) to reduce the 497 difficulty.

\section{$498 \quad$ 3.1.3 Truncated hierarchically organized surfaces}

499 In these cases, there are hierarchically organized surfaces that should be truncated against each other to 500 make watertight subvolumes (surfaces that terminate on the body of another surface, e.g., clinoform 501 surfaces). Jacquemyn et al. 2019 gave instructions for building such topologies with NURBS (e.g., model 502 from higher hierarchal levels to lower hierarchal levels because the coordinates of lower levels are relative 503 to higher levels; then, perform the termination operation) (Fig. 23a). However, referring to several related 504 computer graphics sources, such as (Urick et al. 2019, Pungotra et al. 2010, Sederberg et al. 2008, Sederberg 505 et al. 2003, Chui et al. 2000), reveals that using NURBS for modeling such complex structures is 506 challenging because of the undesirable gaps arising at the boundaries between surfaces. Generally, the 507 inherent difficulties associated with NURBS surfaces, such as limitations in stitching and difficulties in 508 trimming the surfaces for building watertight volumes, complicate the whole modeling process.

509 Based on the subdivision surfaces algorithm, first, a simple watertight layered topology is defined (Fig. 510 23b). Next, the crease sharpness value of each edge is specified, and finally, since such complex topologies

511 are considered "non-manifold topologies", the subdivision approach is compatible with non-manifold 512 topologies applied to this topology. 


\section{a}

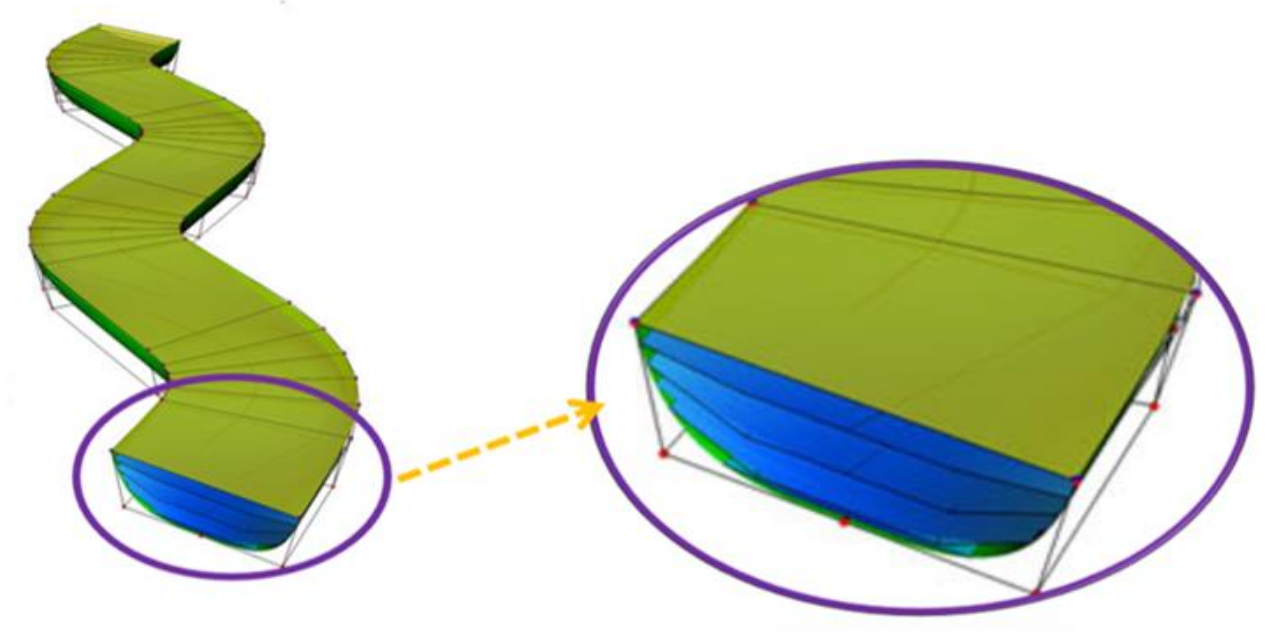

b
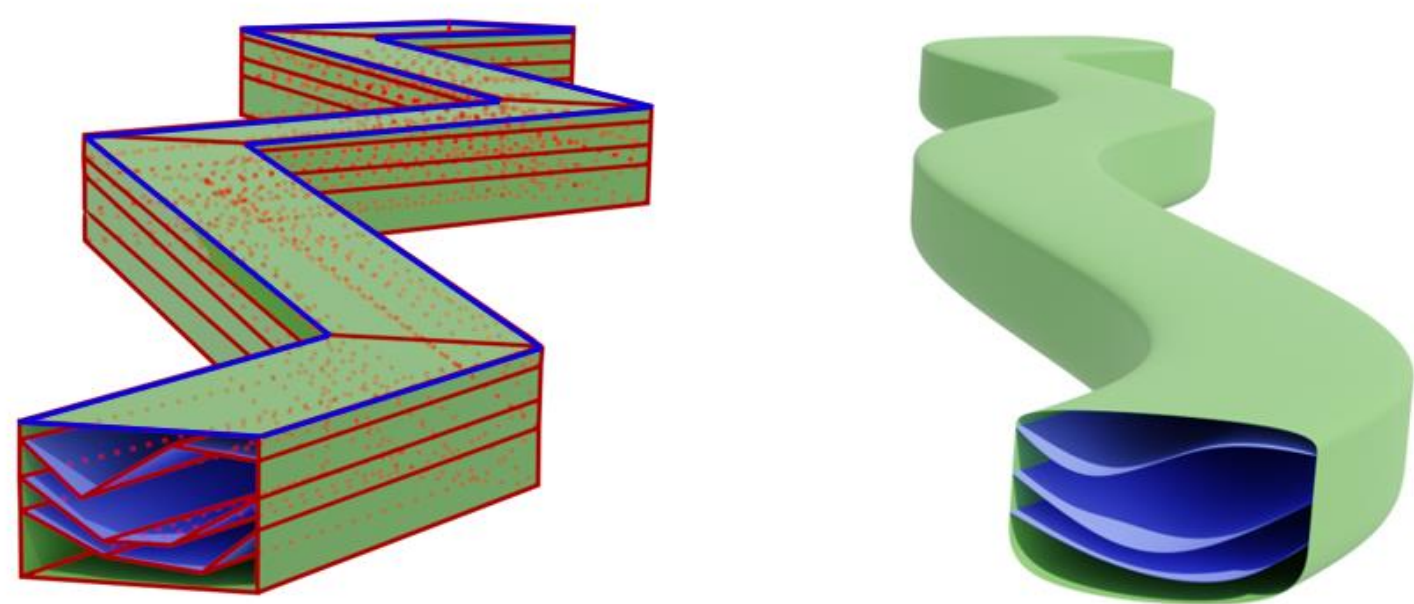

Fig. 23 a Termination of hierarchically arranged surfaces by NURBS for the basal surface of a channelized body (Jacquemyn et al. 2019). b Applying non-manifold Subdivision surfaces to create hierarchically arranged surfaces.

520 As mentioned in section 3.2, NURBS support "non-uniform" parametrization by using the knot vector, 521 which can change the continuity (degree) of the curve or surface at any knot (Ruiu et al. 2016, Cashman 522 2010). The classic subdivision scheme cannot support "non-uniform" parametrization. However, it provides 
523 a significant benefit over NURBS by supporting watertight surfaces with arbitrary topology since it 524 eliminates the procedure of stitching and editing different surface patches (Cashman 2010). There are 525 solutions (methods) that exploit the advantages of both NURBS and subdivision schemes, e.g., NURBS 526 compatible with subdivision surfaces (Cashman 2010), Non-uniform recursive subdivision Surfaces 527 (Sederberg et al. 1998) and T-NURCCs (Non-Uniform Rational Catmull-Clark Surfaces with T-junctions) 528 (Sederberg et al. 2003).

529 In reservoir modeling, NURBS "curves" have been used to represent well trajectories (Jacquemyn et al. 530 2019). Additionally, NURBS "surfaces" have been used for modeling sinuous channels by tensor products 531 between two NURBS curves: one NURBS curve for defining the cross-section and one curve for the 532 trajectory of the channel (Ruiu et al. 2016). Therefore, Non-uniform parametrization can makes NURBS 533 suitable for modeling structures that have several different meanders (curvatures) along a path (trajectory).

534 On the other hand, subdivision surfaces have fewer difficulties in the procedure of modeling watertight 535 surface intersections, which is more beneficial for channels intersecting with each other or layers. 536 Therefore, using a combination of NURBS and subdivision surfaces for building sinuous channels can be 537 an example of exploiting both methods in reservoir modeling simultaneously (Fig. 24). The NURBS curve 538 is a guideline for the channel trajectory and subdivision surfaces as an arbitrary topology supporter for the 539 cross-section of the channel. 
$\mathbf{a}$

Control points of the NURBS

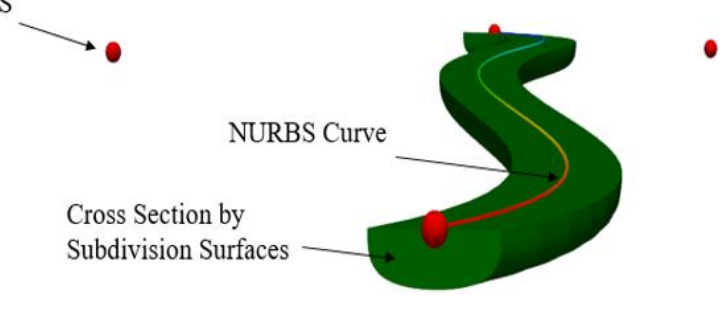

b
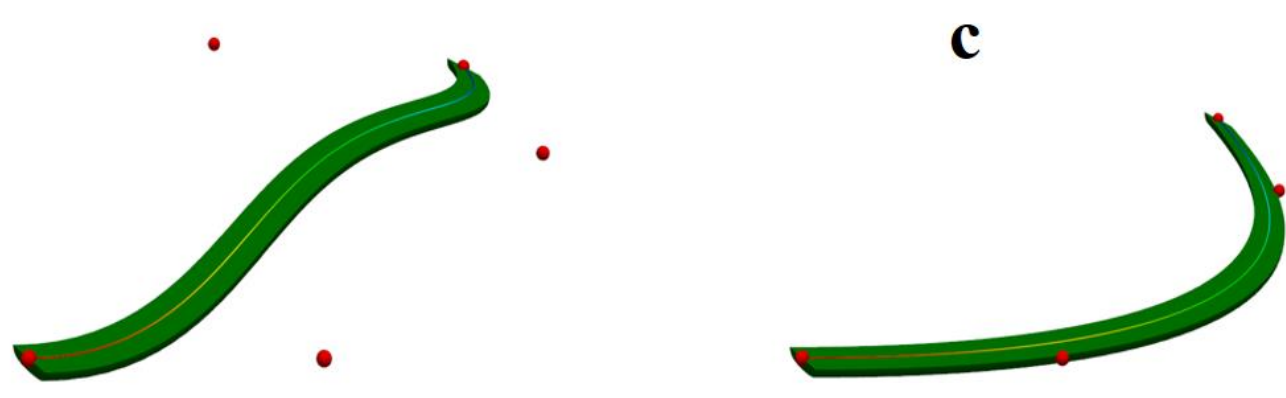

Fig. 24 Using NURBS and subdivision surfaces simultaneously in building channels. a NURBS curve on top of the channel to manage the trajectory of the channel and subdivision surfaces at the cross-section to support the arbitrary and watertight topology of the cross-section. b \& c Different smooth shapes of channels due to different positions of the control points of the NURBS curve.

\section{Approximation of geological and reservoir structures by the parametric surface-based method}

547 Numerical modeling is one of the trustable methods for simulation of the geological process since it can 548 satisfy the mechanical equilibrium equations (Barnichon 1988). Also, mesh density plays an important role 549 in the accuracy and cost of numerical modeling. Ma et al. 2015 proposed to use the simplified 550 (approximated) models of the dense meshes which are made by fitting smoothly controllable surfaces 551 (parametric surface-based models), to make the procedure of processing easier. They mentioned that 552 NURBS and Subdivision surfaces are commonly used to fit parametric models with mesh or dense data;

553 however, NURBS are primarily used for approximating topologically simple cases because managing the 554 connections between different patches of NURBS in topologically complex cases is very difficult. 

algorithm:

\subsubsection{First step: Estimation of topology}

558 The initial topology specifies the topological constraints and the local minima or maxima of the surface.

559 Therefore, estimating the topology is an important step of the approximation procedure. The input data

560 (geological and reservoir structures) can be represented as a mesh (e.g., extracted by software) or as point clouds (e.g., extracted from the structure by the motion technique).

562 Estellers et al. 2018 explained different ways for the estimation of topology when the input data is a mesh or point clouds. They mentioned when the input data is a mesh, using mesh simplification methods (e.g.,

564 "quadratic edge collapse decimation" (Garland and Heckbert 1997)) while maintaining the topology can

565 give an acceptable estimation of topology for approximation. Therefore, the first estimated mesh will have 566 an identical topology to the original mesh while having fewer vertices.

567 Also, they proposed to extract the mesh by using implicit representation for the estimation of the initial 568 topology when the input data is point cloud. Now we can reduce the vertices of this mesh while preserving 569 the topology of it by "quadratic edge collapse decimation" (similar to the steps for input data represented 570 as a mesh).

571 At this step, since the simplified mesh has less complexity, it is possible to make it watertight by adding 572 control points at the intersection places (e.g. intersection between two faults, faults and other layers or two 573 layers).

$574 \quad$ 5.1.2 Second step: Assigning crease sharpness value to each edge and applying subdivision surfaces 575 algorithm to the model

576 In order to define smooth parts of the model (e.g. folds) unique crease sharpness value should be assigned 577 to each edge of the estimated topology (initial model) and then the non-manifold subdivision surfaces 578 algorithm applied to the mesh to perform local or global smoothing.

\subsubsection{Third step: Approximation of the original mesh}

580 Based on computer graphics references, minimizing the sum of the squared distances between the vertices 581 of the original mesh and the approximated mesh is a common approach for approximating a mesh (Jaimez et al. 2017, Hoppe et al. 1994). However, several previous works have developed this approach and made 
$\mathrm{E}(S)=\operatorname{Distance}(S, \mathrm{P})+\alpha * \operatorname{Tan}(S, \mathrm{P})+\beta^{*} R(S)$,

$\operatorname{Distance}(S, \mathrm{P})=\sum_{j=1}^{N} \min \left|\left(S-P_{j}\right)\right|$,

Additionally, all the boundary points of the input mesh $B=\left\{p_{1}, . ., p_{M}\right\}$ should be mapped to the corresponding points of the final surface.

The second part of equation 13 is tangent fitting using the Tan function (equation 15). Fig. 25 shows the tangent surface of point $\mathrm{X}$ of surface $s$ (approximated surface), which includes two orthogonal vectors ( $\eta$ and $\xi$ ). In the best scenario of fitting the surface to the input mesh, the normal of the input mesh should be perpendicular to the tangent surface and naturally perpendicular to $\eta$ and $\xi$ separately. Therefore, the mathematical inner product of the normal vector of the point and each of the two vectors should be minimized (it should be zero in the best scenario).

$\operatorname{Tan}(S, \mathrm{P})=\sum_{j=1}^{N}\left|t_{j} \cdot \eta\right|+\left|t_{j} \cdot \xi\right|$ 


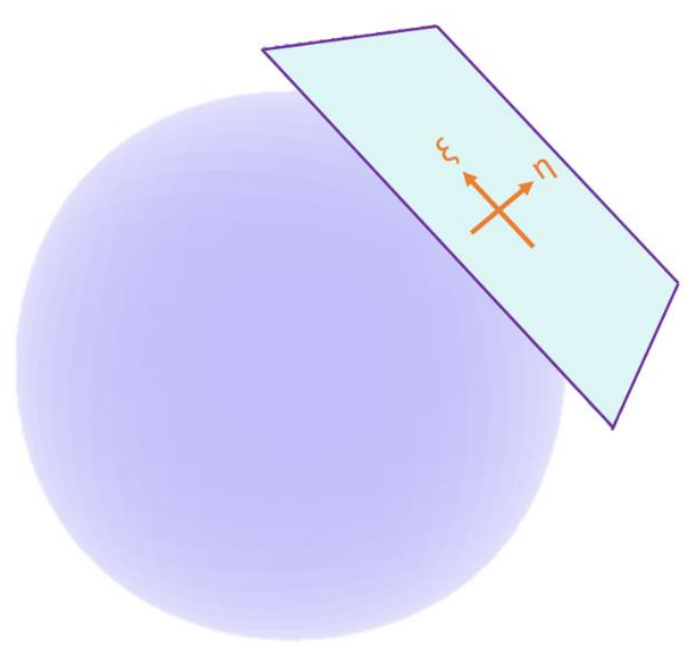

Fig. 25 Tangent surface (Huang et al. 2017).

612 The third part of equation 13 is called regularization $\mathrm{R}(S)$, which is mostly an attempt to avoid the creation 613 of nonstandard elements, e.g., skewed elements. The distance between the vertices of the control mesh in 614 the same quadrant is regularized by equation 16. $R$ is a sparse matrix that consists of all vertices in the 615 columns and edges in the rows. For each edge e that is topologically connected to vertices $v_{1}$ and $v_{2}$, the 616 arrays of $R$ corresponding to the $\left(\mathrm{e}, \mathrm{v}_{l}\right)$ and $\left(\mathrm{e}, \mathrm{v}_{2}\right)$ matrices are 1 and -1 , respectively. $v$ is the matrix of the vertices consisting of the locations of the vertices in each column and the indices of the vertices in each row.

\subsection{Case Studies for the Approximation of Geological Structures}

622 to illustrate the workflow, two geological structures, a folded domain with unconformity and a fault and the 623 Perth Basin geothermal resource (Australia), are approximated. In all cases, the initial simple and watertight 624 topologies are prepared based on section 5.1.1. In the second step, the initial models are modified by 625 applying the subdivision surfaces algorithm based on the crease sharpness value for each edge (manually assigned), and finally, the model is approximated by equation 13. The examples have been generated by the python package GempyExplicit software. GempyExplicit is an open-source Python library for explicit modeling. It can generate both spline and subdivision surfaces. 


\subsubsection{A Folded Domain with Unconformity and a Fault}

631 A folded domain with unconformity and a fault is a conceptual model. This model consists of two important 632 features: smooth surfaces and faults. First, the original mesh based on the real data is generated by Gempy 633 software (De la Varga et al. 2019) (Fig. 26a).

634 In this case, the original mesh has 26000 vertices. Based on section 5.1.1, the first control mesh generated 635 by using a "quadratic edge collapse decimation method" (decrease the number of vertices of original mesh 636 from 26000 to 56) (Fig 26b). Therefore, the control mesh has 56 control points, which are mostly on the 637 intersecting parts of the models, e.g., the intersections between layers and faults to make the model 638 watertight. Also, crease sharpness values are assigned to the edges. The crease sharpness values for the 639 edges that should create smooth surfaces (red edges) are zero, and for the edges that should be sharp (blue 640 edges), are ten. Finally, the non-manifold subdivision surfaces algorithm is applied two times to the control 641 mesh to generate a final mesh (Fig 26c). The final mesh has 1187 vertices (approximately $5 \%$ of the vertices 642 in the original mesh).

643 644 645 646 647 648 649 650 651 
Preprint- Moulaeifard et al. (Submitted to Mathematical Geoscince)

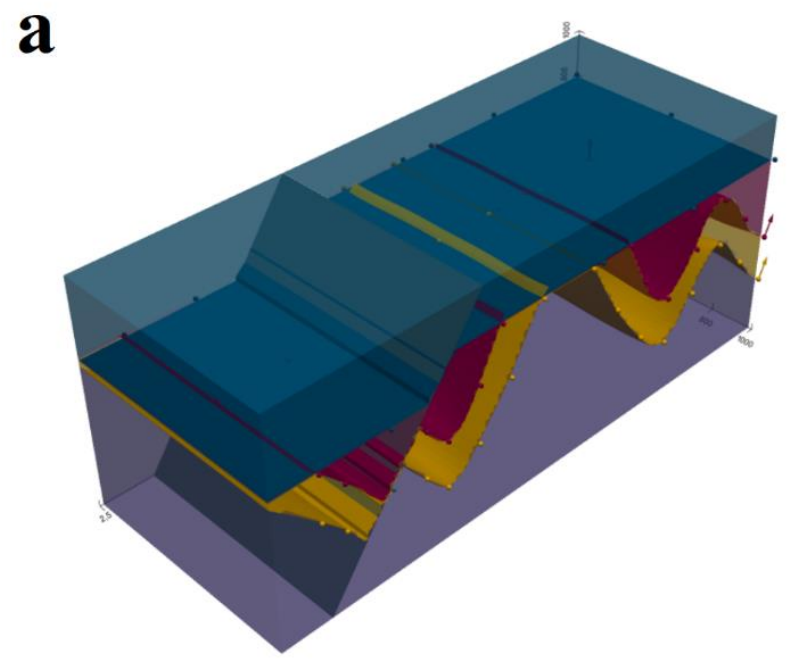

b

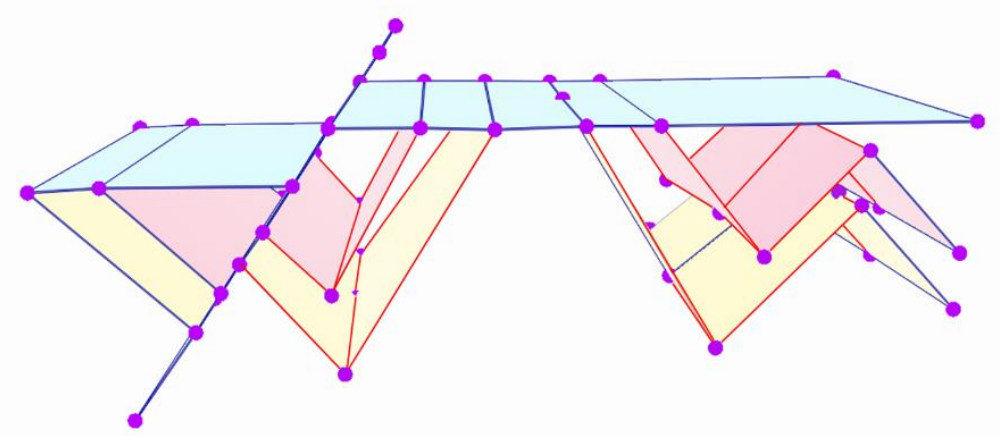

656

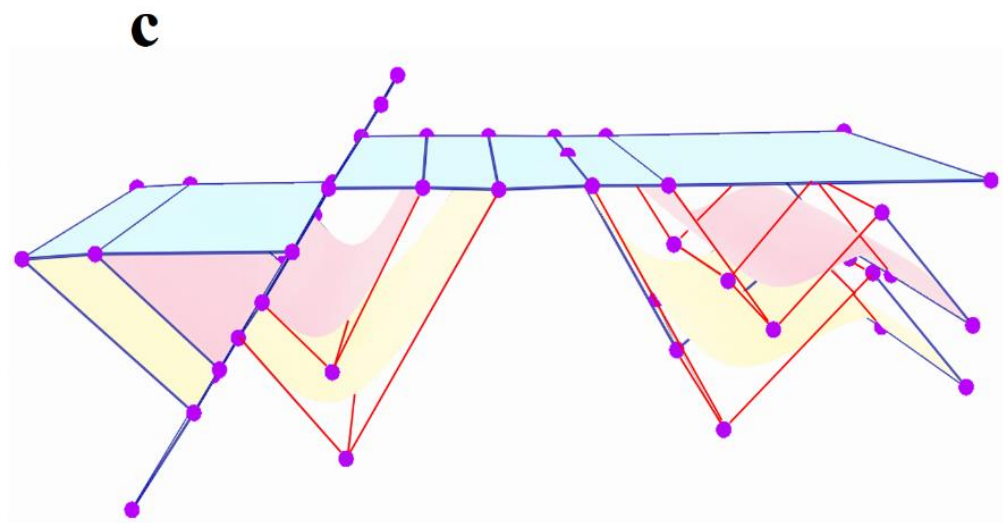

658 Fig. 26 a Gempy (original) model with approximately 26000 vertices. b Watertight and smooth approximated model with 56 control points. The blue and red edges have associated crease sharpness values of ten and zero, respectively. c the final model with 1187 vertices, generated after applying two times subdivision algorithm. 


\subsubsection{Model of Perth Basin, Australia (Geothermal Resource)}

Perth Basin is a long geological rift on the southwestern margin of Australia that contains hydrothermal energy resources. Perth Basin consists of several faults that make it complicated from a modeling point of view. Simulation and modeling of Perth Basin have been investigated in several works (Wellmann and Reid 2014, Olierook et al. 2015, Niederau et al. 2017, De la Varga et al. 2019). Similar to the previous case study, first, the original mesh based on the real data is generated by Gempy software (Fig. 27), and the

667 control mesh (first estimated mesh) is generated by the quadratic edge collapse decimation method while 668 the topology is preserved (section 5.1.1).

669 There are two important points regarding the original model of Perth Basin that make the approximation 670 more complicated. First, the original model has a large number of vertices (approximately 182000 vertices), 671 which shows that many details should be kept and considered during the process of approximation. Second, 672 the original model is not watertight, so several faults and their intersections with layers or other faults make 673 the modeling process frustratingly difficult.

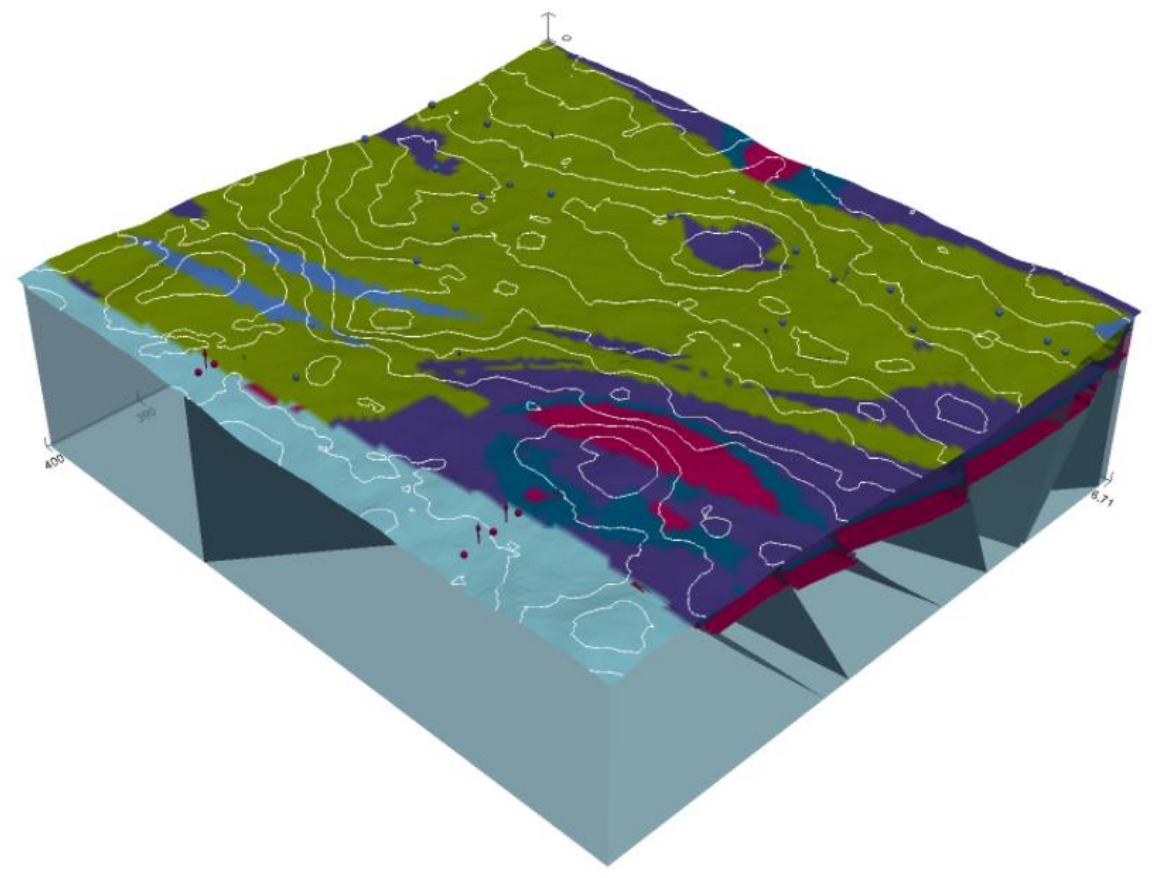

675 Fig. 27 The initial Perth Basin 3D model built by Gempy software (www.gempy.org), with approximately 182000 vertices. 
Preprint- Moulaeifard et al. (Submitted to Mathematical Geoscince)

677 Fig. 28 shows the watertight smooth approximated model. The final approximated model has multiple 678 nodes at intersection points between two faults or between faults and layers that make the model watertight. 679 Additionally, the approximated model has approximately 480 control points and 7645 vertices, which seems 680 to be a large number at first glance, but considering the large number of vertices in the original model 681 (approximately 182000 vertices) and referring to related computer graphics papers, e.g., Estellers et al. 682 (2018), the number of vertices can be acceptable when it is less than 5\% of the number of vertices in the 683 original mesh.

a

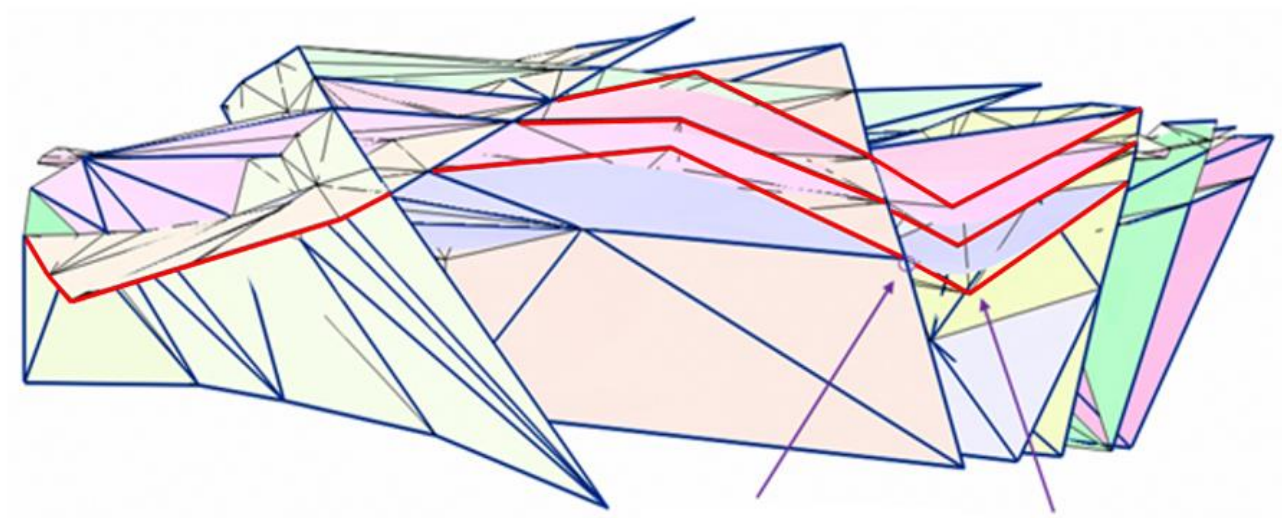

An example of an intersection point for making a watertight model

An example of a smooth place made by edges with zero crease values

b

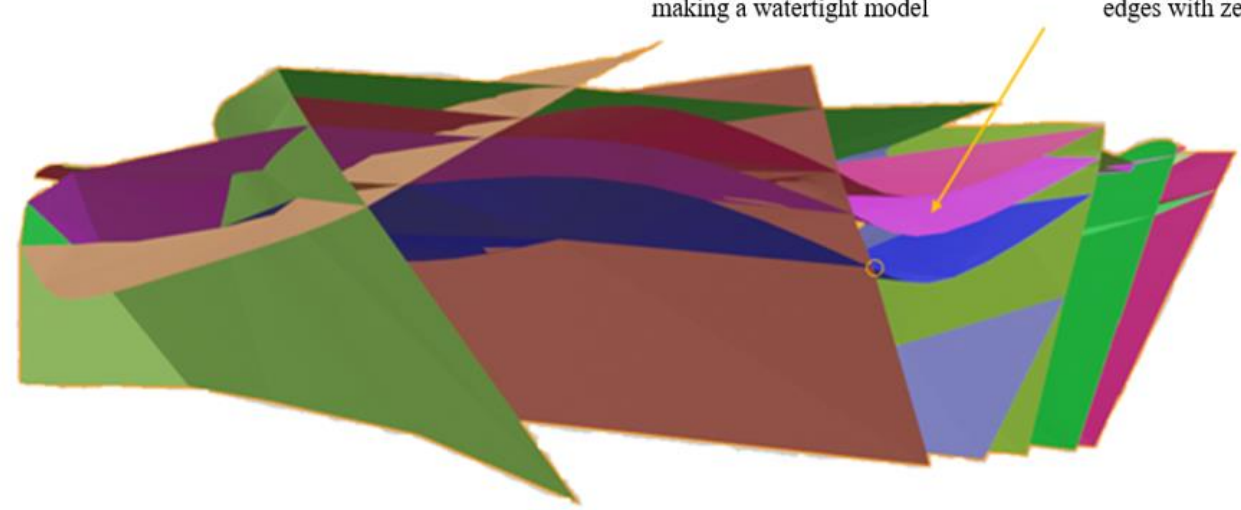

Fig. 28 Watertight approximated Perth Basin model with 480 control points. a An approximated model with nodes at each intersection points and different crease sharpness values for each edge (the bold black and blue edges have crease edge values of zero and ten, respectively). b Final watertight and smooth approximation of the Perth Basin. 


\section{Conclusion}

691 Investigating computer graphics achievements can not only provide insights and bring ingenuity into 692 complex geological modeling but also help to identify common mistakes and develop problem-solving 693 strategies in geological and reservoir modeling. In this paper, NURBS and subdivision surfaces, as two 694 main parametric surface-based representation methods in computer graphics and geological modeling, were 695 discussed. NURBS surfaces have become a standard method in CAD and have been used in explicit geological and reservoir modeling in several works. Subdivision surfaces are a popular method in the animation and gaming industry and are rarely used in geological and reservoir modeling. In the modeling of a complex structure, using NURBS is problematic because it requires a regular gridded structure and several patches; therefore, special care needs to be taken in stitching and trimming. However, subdivision surfaces address these concerns by supporting arbitrary topological structures and making seamless models. Additionally, the subdivision surface method has the ability of local modification, which is difficult in classical NURBS. Understanding the similarities and differences of parametric surface-based models from a computer graphics point of view can help geologists make better decision making during complex geological modelling.

In this paper, the concept of non-manifold topology in geological and reservoir modeling was scrutinized. Classic subdivision scheme cannot represent non-manifold structures since these structures require more complex algorithms. Therefore, the subdivision surfaces compatible with non-manifold topologies were investigated. Additionally, subdivision surfaces were used to approximate the complex geological models examined. The approximated model is not only topologically identical to the geological structure with few control points but also benefits from subdivision surfaces advantages; e.g., it is smooth, controllable and watertight. Using the approximated models gives more control over the model and reduces the number of vertices (to less than $5 \%$ of the number of vertices in the original mesh).

Acknowledgements We would like to thank our colleagues whose previous works motivated us to work on the topic especially, Prof. Guillaume Caumon, Dr. Pauline Collon, Prof. Leif Kobbelt, Prof. Mathew Jackson. This project has agreements No 16258 and No 19004. 
Preprint- Moulaeifard et al. (Submitted to Mathematical Geoscince)

\section{References}

721 Barnichon, J. D. (1998). Finite element modelling in structural and petroleum geology (Doctoral dissertation, 722 Universite de Liege).

723 Botsch, M., Kobbelt, L., Pauly, M., Alliez, P., \& Lévy, B. (2010). Polygon mesh processing. CRC press.

724 Börner, J. H., Bär, M., \& Spitzer, K. (2015). Electromagnetic methods for exploration and monitoring of enhanced 725 geothermal systems-a virtual experiment. Geothermics, 55, 78-87. Cashman, T. J. (2010). NURBS-compatible subdivision surfaces (Doctoral dissertation, Cashman, Thomas J.).

727 Catmull, E., \& Clark, J. (1978). Recursively generated B-spline surfaces on arbitrary topological meshes. Computer728 aided design, 10(6), 350-355.

729 Caumon, G., Lepage, F., Sword, C. H., \& Mallet, J. L. (2004). Building and editing a sealed geological model. 730 Mathematical Geology, 36(4), 405-424.

731 Caumon, G., Collon-Drouaillet, P. L. C. D., De Veslud, C. L. C., Viseur, S., \& Sausse, J. (2009). Surface-based 3D 732 modeling of geological structures. Mathematical Geosciences, 41(8), 927-945.

733 Chatzivasileiadi, A., Wardhana, N. M., Jabi, W., Aish, R., \& Lannon, S. (2018). Characteristics of 3D solid modeling 734 software libraries for non-manifold modeling. Computer-Aided Design and Applications, 16(3), 496-518.

735 Chen, L. Q., \& Liu, D. (2012). Research on the three-dimensional geological modeling based on subdivision surface 736 modeling technology. In Key Engineering Materials (Vol. 500, pp. 646-651). Trans Tech Publications Ltd.

737 Chui, C. K., Lai, M. J., \& Lian, J. A. (2000). Algorithms for G 1 connection of multiple parametric bicubic NURBS 738 surfaces. Numerical Algorithms, 23(4), 285-313.

739 Community, B. O. (2018). Blender - a 3D modelling and rendering package. Stichting Blender Foundation, 740 Amsterdam. Retrieved from http://www.blender.org

741 Corbett, P. W. M., Geiger, S., Borges, L., Garayev, M., \& Valdez, C. (2012). The third porosity system understanding 742 the role of hidden pore systems in well-test interpretation in carbonates. Petroleum Geoscience, 18(1), 73-81.

743 Dassi, F., Perotto, S., Formaggia, L., \& Ruffo, P. (2014). Efficient geometric reconstruction of complex geological 744 structures. Mathematics and Computers in Simulation, 106, 163-184.

745 De Kemp, E. A. (1999). Visualization of complex geological structures using 3-D Bezier construction tools. 746 Computers \& Geosciences, 25(5), 581-597. 
Preprint- Moulaeifard et al. (Submitted to Mathematical Geoscince)

747

DeRose, T., Kass, M., \& Truong, T. (1998, July). Subdivision surfaces in character animation. In Proceedings of the 25th annual conference on Computer graphics and interactive techniques (pp. 85-94).

Deveugle, P. E., Jackson, M. D., Hampson, G. J., Farrell, M. E., Sprague, A. R., Stewart, J., \& Calvert, C. S. (2011). Characterization of stratigraphic architecture and its impact on fluid flow in a fluvial-dominated deltaic reservoir analog: Upper Cretaceous Ferron Sandstone Member, Utah. AAPG bulletin, 95(5), 693-727.

Doo, D., \& Sabin, M. (1978). Behaviour of recursive division surfaces near extraordinary points. Computer-Aided Design, 10(6), 356-360.

Estellers, V., Schmidt, F., \& Cremers, D. (2018, September). Robust Fitting of Subdivision Surfaces for Smooth Shape Analysis. In 2018 International Conference on 3D Vision (3DV) (pp. 277-285). IEEE.

Fabri, A., \& Pion, S. (2009, November). CGAL: The computational geometry algorithms library. In Proceedings of the 17th ACM SIGSPATIAL international conference on advances in geographic information systems (pp. 538-539).

Florez, H., Manzanilla-Morillo, R., Florez, J., \& Wheeler, M. F. (2014). Spline-based reservoir's geometry reconstruction and mesh generation for coupled flow and mechanics simulation. Computational Geosciences, 18(6), 949-967.

Garland, M., \& Heckbert, P. S. (1997, August). Surface simplification using quadric error metrics. In Proceedings of the 24th annual conference on Computer graphics and interactive techniques (pp. 209-216).

Geiger, S., \& Matthäi, S. (2014). What can we learn from high-resolution numerical simulations of single-and multiphase fluid flow in fractured outcrop analogues?. Geological Society, London, Special Publications, 374(1), 125-144.

Graham, G. H., Jackson, M. D., \& Hampson, G. J. (2015). Three-dimensional modeling of clinoforms in shallowmarine reservoirs: Part 1. Concepts and application. AAPG Bulletin, 99(6), 1013-1047.

Hassanpour, M. M., Pyrcz, M. J., \& Deutsch, C. V. (2013). Improved geostatistical models of inclined heterolithic strata for McMurray Formation, Alberta, Canada. AAPG bulletin, 97(7), 1209-1224.

Hoppe, H., DeRose, T., Duchamp, T., Halstead, M., Jin, H., McDonald, J., ... \& Stuetzle, W. (1994, July). Piecewise smooth surface reconstruction. In Proceedings of the 21st annual conference on Computer graphics and interactive techniques (pp. 295-302).

Huang, W., Absil, P. A., \& Gallivan, K. A. (2017). Intrinsic representation of tangent vectors and vector transports on matrix manifolds. Numerische Mathematik, 136(2), 523-543.

Jackson, M. D., Hampson, G. J., Saunders, J. H., El-Sheikh, A., Graham, G. H., \& Massart, B. Y. G. (2014). Surfacebased reservoir modelling for flow simulation. Geological Society, London, Special Publications, 387(1), 271-292. 
Preprint- Moulaeifard et al. (Submitted to Mathematical Geoscince)

776

777

778

779

Jacquemyn, C., Melnikova, Y., Jackson, M. D., Hampson, G. J., \& John, C. M. (2016, August). Geologic modelling using parametric nurbs surfaces. In ECMOR XV-15th European Conference on the Mathematics of Oil Recovery (pp. cp-494). European Association of Geoscientists \& Engineers.

Jacquemyn, C., Jackson, M. D., \& Hampson, G. J. (2019). Surface-based geological reservoir modelling using gridfree NURBS curves and surfaces. Mathematical Geosciences, 51(1), 1-28.

Jaimez, M., Kerl, C., Gonzalez-Jimenez, J., \& Cremers, D. (2017, May). Fast odometry and scene flow from RGB-D cameras based on geometric clustering. In 2017 IEEE International Conference on Robotics and Automation (ICRA) (pp. 3992-3999). IEEE.

Kobbelt, L., Campagna, S., \& Seidel, H. P. (1998, June). A general framework for mesh decimation. In Graphics interface (Vol. 98, pp. 43-50).

Loop, C. (1987). Smooth subdivision surfaces based on triangles. Master's thesis, University of Utah, Department of Mathematics.

Ma, X., Keates, S., Jiang, Y., \& Kosinka, J. (2015). Subdivision surface fitting to a dense mesh using ridges and umbilics. Computer Aided Geometric Design, 32, 5-21.

Niederau, J., Ebigbo, A., Marquart, G., Arnold, J., \& Clauser, C. (2017). On the impact of spatially heterogenous permeability on free convection in the Perth Basin, Australia. Geothermics, 66, 119-133.

Olierook, H. K. H., Timms, N. E., Wellmann, J. F., Corbel, S., \& Wilkes, P. G. (2015). 3D structural and stratigraphic model of the Perth Basin, Western Australia: Implications for sub-basin evolution. Australian Journal of Earth Sciences, 62(4), 447-467.

Paluszny, A., Matthäi, S. K., \& Hohmeyer, M. (2007). Hybrid finite element-finite volume discretization of complex geologic structures and a new simulation workflow demonstrated on fractured rocks. Geofluids, 7(2), 186-208.

Piegl L, Tiller W (1997) The NURBS book. Monographs in visual communications, 2nd edn. Springer,

London.

Pixar Graphics Technologies. Http://graphics.pixar.com/

Pungotra, H., Knopf, G. K., \& Canas, R. (2010). Merging multiple B-spline surface patches in a virtual reality environment. Computer-Aided Design, 42(10), 847-859.

Pyrcz, M. J., Boisvert, J. B., \& Deutsch, C. V. (2009). ALLUVSIM: A program for event-based stochastic modeling of fluvial depositional systems. Computers \& Geosciences, 35(8), 1671-1685. 
Preprint- Moulaeifard et al. (Submitted to Mathematical Geoscince)

Rossignac, J., \& Cardoze, D. (1999, June). Matchmaker: Manifold Breps for non-manifold r-sets. In Proceedings of the fifth ACM symposium on Solid modeling and applications (pp. 31-41).

Ruiu, J., Caumon, G., \& Viseur, S. (2016). Modeling channel forms and related sedimentary objects using a boundary representation based on non-uniform rational B-splines. Mathematical Geosciences, 48(3), 259-284.

Sederberg, T. W. (1985). Piecewise algebraic surface patches. Computer Aided Geometric Design, 2(1-3), 53-59.

Sederberg, T. W., Zheng, J., Bakenov, A., \& Nasri, A. (2003). T-splines and T-NURCCs. ACM transactions on graphics (TOG), 22(3), 477-484.

Sederberg, T. W., Cardon, D. L., Finnigan, G. T., North, N. S., Zheng, J., \& Lyche, T. (2004). T-spline simplification and local refinement. ACM transactions on graphics (TOG), 23(3), 276-283.

Sederberg, T. W., Finnigan, G. T., Li, X., Lin, H., \& Ipson, H. (2008). Watertight trimmed NURBS. ACM Transactions on Graphics (TOG), 27(3), 1-8.

Shen, J., Kosinka, J., Sabin, M. A., \& Dodgson, N. A. (2014). Conversion of trimmed NURBS surfaces to CatmullClark subdivision surfaces. Computer Aided Geometric Design, 31(7-8), 486-498.

Thiele, S. T., Jessell, M. W., Lindsay, M., Ogarko, V., Wellmann, J. F., \& Pakyuz-Charrier, E. (2016). The topology of geology 1: Topological analysis. Journal of Structural Geology, 91, 27-38.

Urick, B., Marussig, B., Cohen, E., Crawford, R. H., Hughes, T. J., \& Riesenfeld, R. F. (2019). Watertight Boolean operations: A framework for creating CAD-compatible gap-free editable solid models. Computer-aided design, 115, 147-160.

de la Varga, M., Schaaf, A., \& Wellmann, F. (2019). GemPy 1.0: open-source stochastic geological modeling and inversion. Geoscientific Model Development.

Villemin, R., Hery, C., Konishi, S., Tejima, T., Villemin, R., \& Yu, D. G. (2015). Art and technology at Pixar, from Toy Story to today. In SIGGRAPH Asia 2015 Courses (pp. 1-89).

Watlet, A., Triantafyllou, A., Kaufmann, O., \& Le Mouelic, S. (2016). Exploring structures of the Rochefort Cave (Belgium) with 3D models from LIDAR scans and UAV photoscans. AGUFM, 2016, T41A-2897.

Wellmann, F., \& Caumon, G. (2018). 3-D Structural geological models: Concepts, methods, and uncertainties. In Advances in Geophysics (Vol. 59, pp. 1-121). Elsevier.

Wellmann, J. F., \& Reid, L. B. (2014). Basin-scale geothermal model calibration: Experience from the Perth Basin, Australia. Energy Procedia, 59, 382-389. 
Preprint- Moulaeifard et al. (Submitted to Mathematical Geoscince)

832 Ying, L., \& Zorin, D. (2001, October). Nonmanifold subdivision. In Proceedings Visualization, 2001. VIS'01. (pp. 833 325-569). IEEE.

834 Zehner, B., Börner, J. H., Görz, I., \& Spitzer, K. (2015). Workflows for generating tetrahedral meshes for finite 835 element simulations on complex geological structures. Computers \& Geosciences, 79, 105-117.

836 Zhang, X., Pyrcz, M. J., \& Deutsch, C. V. (2009). Stochastic surface modeling of deepwater depositional systems for 837 improved reservoir models. Journal of Petroleum Science and Engineering, 68(1-2), 118-134.

838 Zorin, D. (2000). Subdivision for modeling and animation. SIGGRAPH2000 course notes.

839 Zorin, D., \& Schröder, P. (2001). A unified framework for primal/dual quadrilateral subdivision schemes. Computer 840 Aided Geometric Design, 18(5), 429-454.

841 